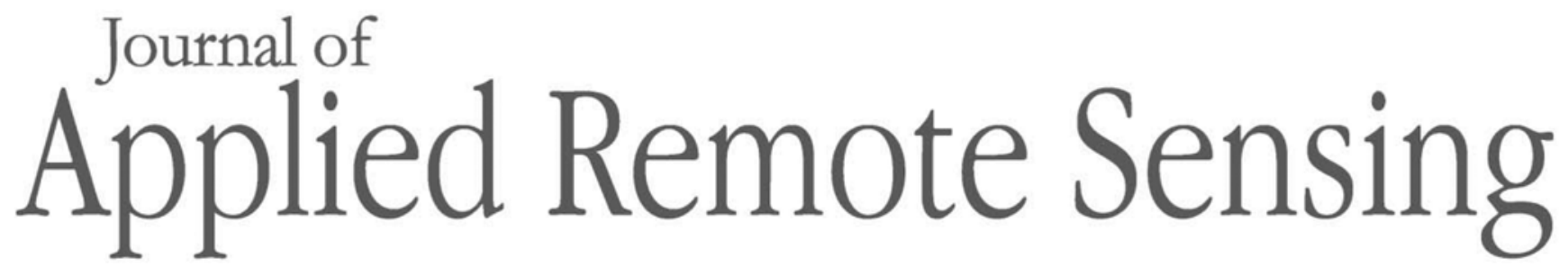

RemoteSensing.SPIEDigitalLibrary.org

\title{
Demonstration of spatial greenhouse gas mapping using laser absorption spectrometers on local scales
}

Jeremy T. Dobler

T. Scott Zaccheo

Timothy G. Pernini

Nathan Blume

Gregoire Broquet

Felix Vogel

Michel Ramonet

Michael Braun

Johannes Staufer

Philippe Ciais

Chris Botos

Jeremy T. Dobler, T. Scott Zaccheo, Timothy G. Pernini, Nathan Blume, Gregoire Broquet, Felix Vogel, Michel Ramonet, Michael Braun, Johannes Staufer, Philippe Ciais, Chris Botos, "Demonstration of spatial 


\title{
Demonstration of spatial greenhouse gas mapping using laser absorption spectrometers on local scales
}

\author{
Jeremy T. Dobler, ${ }^{a}{ }^{a}$ T. Scott Zaccheo, ${ }^{b}$ Timothy G. Pernini, ${ }^{b}$ \\ Nathan Blume, ${ }^{a}$ Gregoire Broquet, ${ }^{c}$ Felix Vogel, ${ }^{c}$ Michel Ramonet, ${ }^{c}$ \\ Michael Braun, ${ }^{a}$ Johannes Staufer, ${ }^{c}$ Philippe Ciais, ${ }^{c}$ and Chris Botos ${ }^{b}$ \\ ${ }^{\mathrm{a}}$ Harris Corporation, Fort Wayne, Indiana, United States \\ ${ }^{\mathrm{b}}$ Atmospheric and Environmental Research Inc., Lexington, Massachusetts, United States \\ ${ }^{c}$ Université Paris-Saclay, Laboratoire des Sciences du Climat et de l'Environnement, \\ LSCE/IPSL, CEA-CNRS-UVSQ, Gif-sur-Yvette, France
}

\begin{abstract}
A system for measuring the two-dimensional (2-D) spatial distribution of atmospheric $\mathrm{CO}_{2}$ over complex industrial sites and urban areas on the order of 1 to $30 \mathrm{~km}^{2}$ every few minutes with a spatial resolution as high as tens of meters has been developed and demonstrated over the past 3 years. The greenhouse gas (GHG) laser imaging tomography experiment (GreenLITE ${ }^{\text {TM}}$ ) provides improved measurement capabilities for applications ranging from automated 24/7 monitoring of ground carbon storage/sequestration (GCS) sites to long-duration real-time analyses of GHG sources and sinks in urban environments. GreenLITE combines a set of sensors based on an intensity modulated continuous wave approach with 2-D sparse tomographic reconstruction mechanisms to compute a 2-D map of $\mathrm{CO}_{2}$ concentrations over the area of interest. GreenLITE systems have recently been deployed at a number of test facilities, including a 4000-h demonstration at a GCS site in Illinois and an urban deployment in Paris, France, from November 2015 to the present. This paper describes the GreenLITE concept and the associated measurement capabilities and provides proof of concept results and analyses of observations from both short-term tests as well as longer-term industrial and urban deployments. () The Authors. Published by SPIE under a Creative Commons Attribution 3.0 Unported License. Distribution or reproduction of this work in whole or in part requires full attribution of the original publication, including its DOI. [DOI: 10.1117/1.JRS.11.014002]
\end{abstract}

Keywords: carbon; laser absorption spectroscopy; tomography; greenhouse gas; carbon storage.

Paper 16701P received Sep. 20, 2016; accepted for publication Feb. 9, 2017; published online Mar. 2, 2017.

\section{Introduction}

The GreenLITE system is designed to provide real-time quantitative measurements of near-surface atmospheric $\mathrm{CO}_{2}$ concentrations in a horizontal plane 1 to $50 \mathrm{~m}$ above ground level (AGL) over large ( 1 to $>30 \mathrm{~km}^{2}$ ) areas with subelement resolution on the order of 100 to $200 \mathrm{~m}^{2}$. This approach provides a unique capability for a number of applications ranging from continuous remote monitoring of ground carbon storage/sequestration (GCS) facilities to the real-time measurement and assessment of subscale greenhouse gas (GHG) events within complex open air environments, e.g., industrial complexes and city sectors. This approach provides a unique perspective in contrast to the current state of the art for GCS atmospheric monitoring, which typically consists of LiDARs, in situ optical sensors, natural and introduced tracers, and eddy covariance $(\mathrm{EC})^{1}$ measurements.

Sensing the distribution of $\mathrm{CO}_{2}$ over GCS sites and urban environments poses a number of challenges. While the objectives in both cases are often to locate and quantify the emissions of temporally and spatially varying sources/sinks distributed across an extended area of interest, the number of source/sink terms, the desired spatial scales, the ambient background terms,

*Address all correspondence to: Jeremy T. Dobler, E-mail: Jeremy.Dobler@harris.com 
geographic extent, and overall objectives tend to be quite different. Often the objectives in a purely industrial environment are the routine monitoring and characterization of the ambient background term and the detection of adverse anomalous conditions over confined areas with a limited set of land cover types, e.g., vegetation and man-made materials. $\mathrm{CO}_{2}$ measurements in urban areas are used in conjunction with transport models (whose horizontal resolution is typically 1 to $4 \mathrm{~km}^{2}$ ) and statistical inversion techniques to estimate city $\mathrm{CO}_{2}$ emissions. ${ }^{2}$ For this objective, the measurement must catch the typical spatial and temporal variations at the scales corresponding to the area (the whole city or neighborhood) targeted by the emission estimate. However, concentrations in urban areas vary widely in spatial scale, have highly complex and temporally varying backgrounds, and have a continuous array of temporally/spatially varying sources and sinks.

In situ optical sensors rely on nondispersive absorption of infrared (IR) radiation, usually in a cavity that is temperature controlled, and often require drying the sample to avoid impacts from atmospheric water vapor. These sensors are commercially available and provide precise [ 0.1 parts per million by volume (ppmv)] measurements of concentration at a single point. They can also provide very accurate data when coupled with appropriate calibration systems using known quantity gas mixtures. Other less expensive sensors are available in exchange for accuracy and precision. Chemical sensors are also available but typically suffering from thermal drifts and contamination from other chemical interactions; they are useful for inexpensive threshold warning systems where only very high concentrations need to be identified for human safety. In situ sensors provide extremely precise measurements at a given point in space, making them ideal for monitoring and quantifying concentrations and emissions for well-defined point sources, e.g., stacks and well heads, as well as large diffuse source terms that are spatially homogeneous over extended areas. However, they are limited in their ability to describe the dynamics of large spatially diverse areas of interest for GCS integrity monitoring or other large-scale applications, e.g., complex urban environments.

Natural and introduced tracers can enhance the ability to detect a GCS leak by marking sequestered $\mathrm{CO}_{2}$ with a tracer. Tracers may make it easier to identify the source of a GCS leak directly versus the naturally varying backgrounds of $\mathrm{CO}_{2}$. However, they often require specialized equipment for evaluating flask samples that are not commonly located at the GCS site. Another challenge for tracer detection is that the tracer compound may behave differently than the $\mathrm{CO}_{2}$ due to interaction with other substances or different diffusion rates in the ground or atmosphere, causing the $\mathrm{CO}_{2}$ release to be misinterpreted.

EC uses a combination of sensors to measure rapidly changing vertical and horizontal winds along with rapidly changing $\mathrm{CO}_{2}$, temperature, humidity, or other trace gases of interest. It employs a fluid dynamics approach where the atmosphere is assumed to be entirely made up of eddies of varying sizes within a horizontal wind flow. ${ }^{3}$ By measuring the up and down parts of the eddy over time, along with the local gas concentration and horizontal flow, one can determine the net flux at the location of the sensor. It relies on two key assumptions: (1) density fluctuations are assumed negligible and (2) mean vertical flow is assumed negligible for horizontal homogeneous terrain. The validity of these assumptions depends on the site location and terrain. EC relies on several other assumptions, and the resulting EC data are complex and hard to quality control and interpret. ${ }^{4}$ It has been shown as a valid approach for determining surface-to-air fluxes over fairly large areas, but the assumptions need to be satisfied, and it is not clear if it can be applied accurately for a wide range of locations and atmospheric conditions. Traditionally, the footprint of an EC measurement is extrapolated using models that combine direct EC tower flux measurements with the horizontal wind flow. ${ }^{5}$

While conventional approaches outlined above provide precision point measurements of $\mathrm{CO}_{2}$ concentrations, and fluxes and large-scale estimates of homogeneous background concentrations, the GreenLITE system, described in Sec. 2, directly measures the $\mathrm{CO}_{2}$ density over the entire area of interest along multiple chords or horizontal columns of air. The data from these overlapping chords are then used in a sparsely sampled tomography approach to estimate the two-dimensional (2-D) distribution of $\mathrm{CO}_{2}$ concentration across the area of interest, as discussed in Sec. 2.2.2. While differencing the 2-D reconstructions as a function of time can provide a first-order estimate of local changes in flux, the current implementation does not include vertical wind speeds and cannot provide absolute flux values. Nor can the current system distinguish 
between vertical fluxes from local sources and sinks or changes in concentrations due to horizontal flow dynamics, although algorithms to do so are in the works. Early work has also been done to use the time varying horizontal wind information, combined with the spatial concentration information, to track and identify plume source location with potential for determining plume strengths. ${ }^{6}$

A number of LiDAR approaches, such as that demonstrated by Repasky et al., ${ }^{7}$ provide close corollaries to the GreenLITE measurement, but GreenLITE is distinctly different in its implementation of intensity modulated continuous wave (IM-CW) laser absorption spectroscopy. While both approaches exploit the differences in atmospheric transmission for two closely spaced, narrow laser lines and benefit from the cancellation of common-mode terms due to other processes (primarily scattering) that are nearly identical at both wavelengths, the other approaches use atmospheric backscatter to provide spatially resolved information. This atmospheric scattering has about 1 million times lower reflectance than the fixed corner cubes used for GreenLITE. This difference causes conventional differential absorption LiDAR (DIAL) systems to be more complex and to require higher laser power, larger optics, and specialty detectors. Although these DIAL instruments showed generally good agreement with independent measurements, most of the testing to date has been performed only in nighttime conditions and showed little, if any, correlation to the temporal variability seen in the in situ measurements. ${ }^{7}$

Recent testing of the GreenLITE system at the Zero Emissions Research and Technology (ZERT) site in Bozeman, Montana, ${ }^{8}$ included similar comparisons of in situ to average GreenLITE $\mathrm{CO}_{2}$ measurements. In this case, the GreenLITE instrument and the in situ instrument showed a correlation coefficient over several hours of $>0.933$ for multiple tests on multiple days, and a correlation coefficient of 0.98 for a 23 -h continuous period. This represents a significant performance improvement in the sensitivity of the GreenLITE approach, which is required for an open-path laser system to detect potential GCS leaks at practical heights above the surface. Prior laser-based open-path measurements, located only $20 \mathrm{~cm}$ above the surface, demonstrated ${ }^{9}$ the ability to detect the signature of controlled ground releases, but may not be practical for monitoring large areas with varying topography, weeds, traffic, and other impediments that mandate the measurement be made several meters above the surface.

While the original GreenLITE system was designed for remote monitoring of GCS sites, similar needs exist for other applications, such as facilities monitoring and urban areas. Currently, tower-based in situ instruments and other point measurements, along with static and dynamic inventories, are being used to help constrain models that provide dynamic estimates of changes in urban concentrations and fluxes. ${ }^{2,10,11}$ Placing towers within the urban environments often presents difficulties due to siting challenges, and point measurements are often adversely impacted by nearby highly localized sources. This susceptibility to local variations presents a significant challenge in assimilating these data into transport models with grid scales on the order of 1 to $4 \mathrm{~km}$. Since the initial deployment of the first GreenLITE system, a second system that directly addresses the urban scale covering areas of $\sim 25 \mathrm{~km}^{2}$ has been deployed in Paris, France. This enables detailed measurements within cities that are not as sensitive to smallscale local variations as current in situ instruments and also provides a wealth of spatial information to better constrain models. Further scaling of the system may potentially support a wider range of applications, such as remote autonomous monitoring of enhanced oil recovery fields. Similar challenges exist for measurements of $\mathrm{CH}_{4}$ at many different source locations from large feedlots and hydraulic fracturing (fracking) fields to underground or offshore storage fields. The GreenLITE system conversion to $\mathrm{CH}_{4}$ is straightforward, mainly requiring changing the laser sources and updating the algorithms for the $\mathrm{CH}_{4}$ spectroscopy, and is currently under development by Harris and Atmospheric and Environmental Research Inc.

In Sec. 2, we describe the hardware, software, and data processing algorithms of the GreenLITE system. In Sec. 3, we describe deployments of the GreenLITE system at controlled test sites, at an active GCS site, and in an urban environment. In Sec. 4, we present analyses and results based on the aforementioned deployments, and in Sec. 5, we summarize our work and offer possibilities for future system improvements and applications. While procedures are in place to calibrate the measurements taken with the GreenLITE system, in particular to address measurement precision and drift, a true reference measurement-and thus bias estimate-are difficult to obtain due to the complexities associated with measuring a 1 to $5 \mathrm{~km}$ nearly 
horizontal column of air in a nonhomogeneous atmosphere that contains spatially varying gaseous mixing ratios, temperatures, and pressures and includes constituents other than the species of interest. Efforts to further improve and validate the precision and accuracy of GreenLITE measurements are ongoing. As such, the deployments and results presented in Secs. 3 and 4 are intended as successful proof of concept endeavors of the GreenLITE system to measure an atmospheric species of interest and to locate potential sources of said species over 2-D areas up to $25 \mathrm{~km}^{2}$.

\section{GreenLITE System}

The GreenLITE system includes both data collection and data processing components. The data collection portion includes transceivers, retroreflectors, and software necessary to operate the transceivers and facilitate data collection. The data processing portion includes algorithms to convert measured differential transmission to integrated $\mathrm{CO}_{2}$ column concentration and estimate 2-D tomographic reconstructions of $\mathrm{CO}_{2}$ concentration fields, as well as a web-based user interface for real-time monitoring. The following two sections provide an overview of these two components.

\subsection{GreenLITE Data Collection}

The GreenLITE sensor is based on the IM-CW approach, ${ }^{12}$ which allows multiple wavelengths of light to be transmitted and received simultaneously. The narrow band lasers are set to transmit light on at least two wavelengths, which correspond to an absorption feature of the molecule to be measured. One laser is placed at a wavelength with high absorption and another laser is placed in a region of lesser absorption. The difference in absorption between the two wavelengths enables the number density of the particular molecule to be determined, assuming the laser and absorption cross-sections are known. The original concept was developed by Dobbs et al. ${ }^{13,14}$ as a method for measuring $\mathrm{CO}_{2}$ sources and sinks from space and led to the development of the multifunctional fiber laser LiDAR (MFLL) as an airborne demonstrator. Since 2005, NASA Langley Research Center has been evaluating the airborne system and has demonstrated that MFLL provides high-accuracy and precision measurements as compared with independent measurements traceable to World Meteorological Organization (WMO) standards. The MFLL instrument has also been selected by NASA Headquarters as a primary instrument for the ongoing Atmospheric Carbon and Transport-America Program, a NASA Earth Venture Suborbital mission.

These efforts have led to the development of a ground-based version of MFLL to demonstrate a concept for making similar measurements in transmission by separating the transmitter and receiver. This configuration can enable a geostationary solution for obtaining high-accuracy and temporally resolved $\mathrm{CO}_{2}$ measurements for targeted observations of vertically integrated column $\mathrm{CO}_{2}$ values not currently offered by any planned or existing space-based remote sensing systems. ${ }^{15}$ The GreenLITE system leveraged the best aspects of these prior works, resulting in a robust and capable design.

The GreenLITE transceiver is divided into two main components: (1) the electro-optical box and (2) the optical head, as shown in Fig. 1. The electro-optical box is a thermally stabilized box that houses the laser transmitters, optical modulators, detector chain for reference and science channels, computer, data acquisition, and other associated electronics. The optical head is also thermally controlled and houses both transmit and receive telescopes. The optical head is mounted on a mechanical scanner that is used to point the optical head at individual retroreflectors. The system is designed to operate in remote locations for long periods of time with minimal interaction.

The current systems operate on an absorption line near $1571.112 \mathrm{~nm}$, with the offline located 50 to $60 \mathrm{pm}$ from the online (nominally $1571.061 \mathrm{~nm}$ ). The 1-km system transmits a total of $5 \mathrm{~mW}$ of optical power combined between online and offline, and the 5-km system produces $\sim 25 \mathrm{~mW}$ of total transmitted power, which is well below the eye-safe limit at this wavelength for the $\sim 20$-mm transmit beam. Both systems use standard InGaAs detectors and have a transmit 


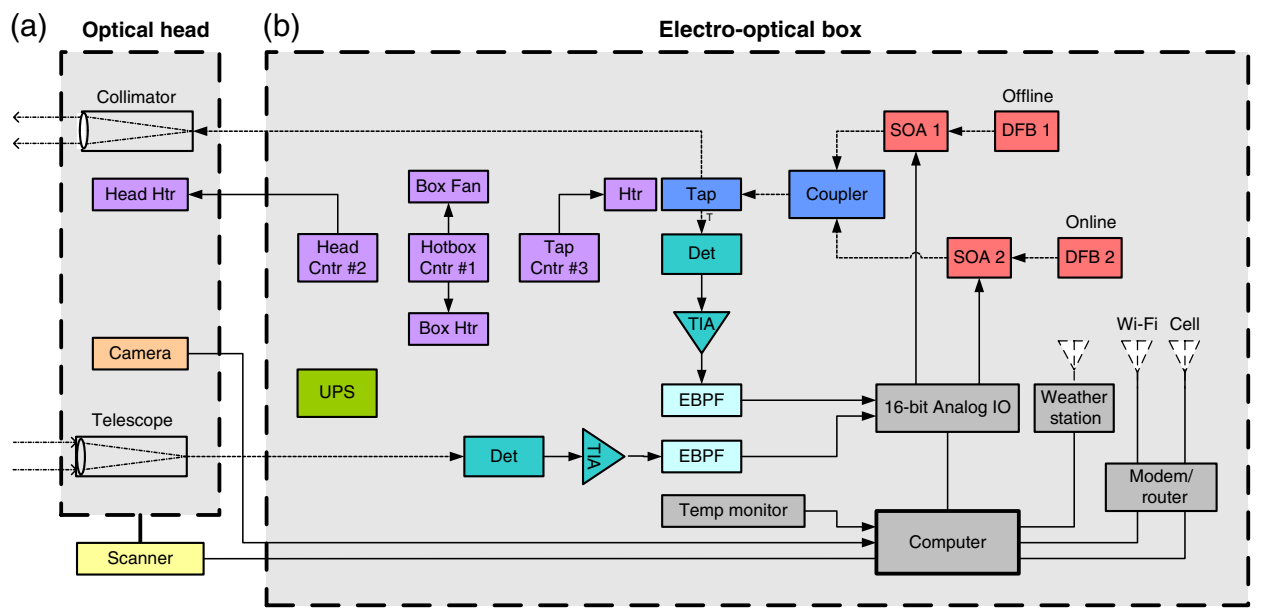

Fig. 1 GreenLITE transceiver block diagram, showing (a) the optical head and (b) the electro-optical box. Acronyms in the figure are as follows: Htr, heater; Cntr, controller; UPS, uninterruptable power supply; Det, detector; TIA, trans impedance amplifier; EBPF, electronic bandpass filter; SOA, semiconductor optical amplifier; DFB, distributed feedback laser; and IO, input/output.

divergence of $1.6 \mathrm{mrad}$, a receiver field of view of $2.6 \mathrm{mrad}$, and a $25.4-\mathrm{mm}$ transmit telescope. The receiver in the 1-km system uses a 25.4-mm diameter F/4 telescope, while the 5-km system uses a 152.4-mm diameter $\mathrm{F} / 2$ telescope.

Two or more transceivers and several retroreflectors are arranged such that the lines (or chords) between the two transceivers and each retroreflector generate a grid of interweaved segments. Each transceiver scans to the retroreflectors in sequence and makes a measurement of each chord for a specific amount of time (typically $10 \mathrm{~s}$ ). After measuring all of the chords, the transceiver makes a zero-path measurement used to evaluate and correct for drift. For the current implementation, the maximum scan angle is $\sim 170 \mathrm{deg}$, limited mainly by cabling. Increasing the scan angle to a full 360-deg range is being evaluated for the next generation. Examples of some of the configurations of the system are shown in Fig. 2. In these examples, the parabolic configuration shown in Fig. 2(c) maximizes the use of the retroreflectors by both transceivers, while covering more area than the more rectilinear configurations shown in the other two examples in Figs. 2(a) and 2(b). Other optimal configurations that maximize the number of intersections over the primary areas of interest are possible, or additional transceivers can be used to optimize coverage for desired shapes depending on the application. The system requires a clear line of sight from the transceivers to the retroreflectors, which can pose challenges. However, the system testing to date has shown that the algorithms discussed in Sec. 3 provide a robust solution

(a)

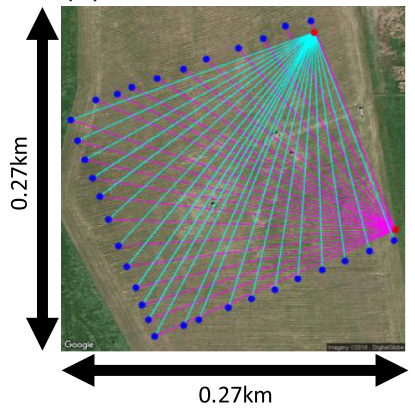

(b)

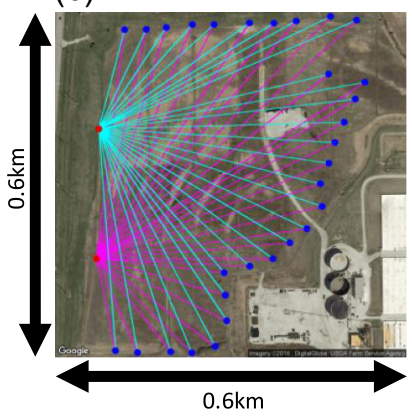

(b)

$0.6 \mathrm{~km}$ (c)

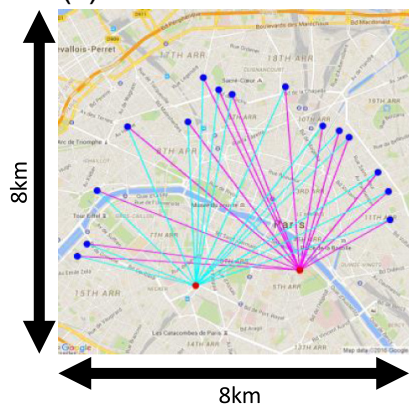

Fig. 2 Example GreenLITE system layouts. (a) A geographic layout of the MSU ZERT site, (b) the GCS test facility in Illinois, and (c) the current GreenLITE deployment in Paris, France. These sites range from $0.27 \mathrm{~km}$ on a side at ZERT to over $8 \mathrm{~km}$ in Paris. The red dots in the images denote the transceiver locations, and the blue dots indicate the placement of 15 to 32 retroreflectors. The cyan and magenta lines depict the lines of sight between each transceiver and the retroreflectors. 
for nonoptimal layouts and sites with a limited number of obstructed chords. This finding enables GreenLITE to be considered for a number of applications where the optimal layout may not be practical, such as an urban environment where the layout is driven by existing buildings and structures suitable for mounting the retroreflectors.

All hardware control and monitoring, data acquisition, preliminary data processing, and data transmission to the remote database are performed by the software running at each transceiver. The transceiver software controls the movement of the mechanical scanner and coordinates the acquisition of data with the scanner movement. The software provides a convenient way to load a list of predefined retroreflector locations. The actual measurement of the differential transmission of the lasers is made using a digital lock-in processing technique. Each laser is intensity modulated at a unique frequency prior to transmission through the atmosphere. A small portion of the laser energy is diverted to a reference detector whose digitized output can be used to normalize the return signal for variations in transmitted power. The collected laser signal returned by the retroreflectors is digitized and mixed with the local oscillators that were used to modulate the lasers and then normalized by the reference signals. This lock-in technique allows very small signals at known frequencies to be detected in the presence of wideband noise many orders of magnitude higher in power than the signals of interest. The normalized online signal is divided by the normalized offline signal to get the differential transmission of the lasers. After each collection period from a retroreflector is complete, the measured differential transmission and all corresponding metadata are formatted and transmitted over the internet to the remote GreenLITE database. This is only a brief description of the instrument as implemented for GreenLITE, and further information on the measurement approach can be found in Ref. 12, 16.

\subsection{GreenLITE Data Processing}

The GreenLITE system computes $\mathrm{CO}_{2}$ densities and near-real-time 2-D maps of $\mathrm{CO}_{2}$ concentrations. Specifically, this process continuously: (1) computes integrated column $\mathrm{CO}_{2}$ concentration (ppmv) from observed differential laser absorption measurements along horizontal lines of sight between a transceiver and a reflectors (chords) and (2) estimates 2-D distribution of timevarying $\mathrm{CO}_{2}$ concentrations based on an analytic plume model and an ensemble set of temporally adjacent integrated chord values. Currently, this process has been implemented in modular fashion where the applications defined above run asynchronously in a multithreaded environment coupled to one another through a common database structure. Near-real-time monitoring of $\mathrm{CO}_{2}$ concentrations and 2-D distribution estimates are made possible through a web-based analysis tool. Additionally, simple temporal differencing of successive 2-D reconstructions can provide a first-order estimate of change across the boundary and, properly combined with concurrent wind information, may be able to directly infer flux.

\subsubsection{Retrieving $\mathrm{CO}_{2}$ concentration from observed optical depth ratios}

The first step in the retrieval process is the conversion of each GreenLITE transceiver's differential transmission measurement to an estimate of the horizontal column $\mathrm{CO}_{2}$ concentration in parts per million (ppmv). This is accomplished by combining the difference in observed "on" and "off" line optical depth values $\left(\Delta \tau=\tau_{\text {on }}-\tau_{\text {off }}\right)$, measured meteorological state, and static path length parameters with a radiative transfer (RT)-based iterative retrieval scheme depicted in Fig. 3. The path lengths are determined using GPS measurements at the transceiver and the reflector to calculate a range and are also directly measured using the phase of the modulated signals. The range uncertainty is $\pm 1 \mathrm{~m}$ and can contribute to the error in the retrieved $\mathrm{CO}_{2}$ by $\pm 0.1 \%$ over a $1-\mathrm{km}$ path length; it would be smaller for longer ranges and higher for shorter ranges.

This algorithm employs a line-by-line RT model, LBLRTM, ${ }^{17,18}$ in conjunction with a standard steepest descent search technique to find the modeled $\Delta \tau$ values that best match the observed $\Delta \tau$ given the atmospheric state along the chord [surface temperature $(T)$, relative humidity $(R H)$, and pressure $(P)]$. The initial algorithm conditions are set to: $(1)$ a fixed estimate of the column concentration, nominally 380 ppmv; (2) the estimated atmospheric state at the 
Dobler et al.: Demonstration of spatial greenhouse gas mapping using laser absorption...

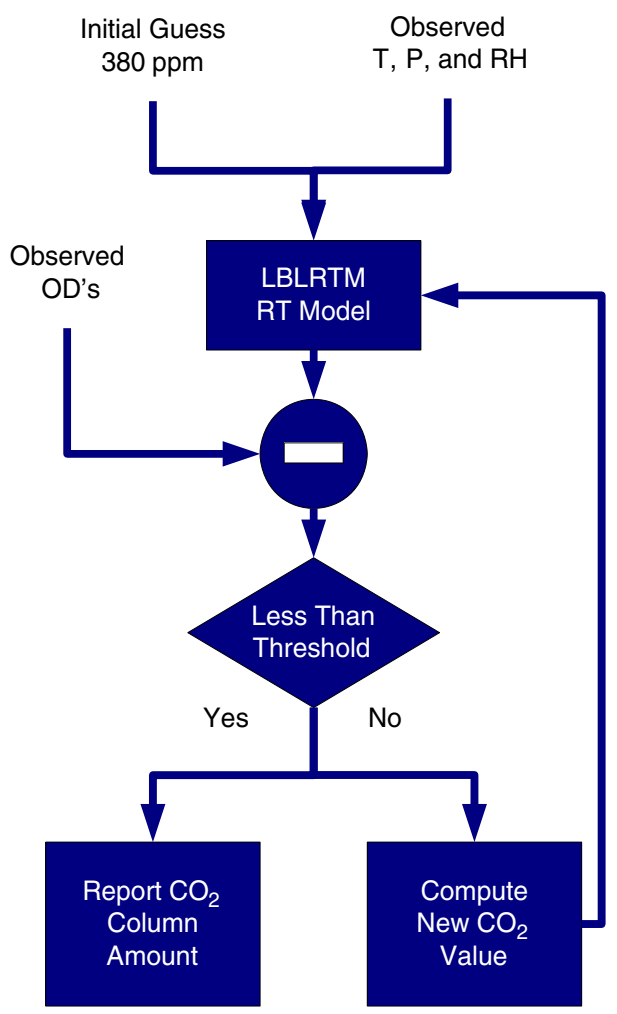

Fig. 3 Iterative flow diagram of $\mathrm{CO}_{2}$ concentration retrieval algorithm.

height of the transceivers and reflectors based on colocated in situ $T / R H / P$ measurements; and (3) static parameters describing the chord geometry. These values are combined in the RT modeling framework to compute a baseline estimate of the modeled differential optical depth

$$
\Delta \tau_{M}=\tau_{M \mathrm{on}}-\tau_{M \mathrm{off}}
$$

where $\tau_{M \text { on }}$ is the model optical depth at the monochromatic online wavelength and $\tau_{M \text { off }}$ is that at the offline wavelength. LBLRTM optical depths are computed from Voigt line shape functions at atmospheric levels and with a continuum model that includes self- and foreign-broadened water vapor, as well as continua for $\mathrm{CO}_{2}, \mathrm{O}_{2}, \mathrm{~N}_{2}$, and $\mathrm{O}_{3}$, and extinction due to Rayleigh scattering. The version used in the work included 2012 updates to the $\mathrm{CO}_{2}$ line parameters and coupling coefficients based on the work of Devi et al. ${ }^{19,20}$

Next, the observed $\Delta \tau$ is compared with the model value $\left(\Delta \tau_{M}\right)$, and the resulting difference is used to update the estimated column concentration. This is achieved by computing a gradient or the change in column concentration as a function of a change in observed optical depth via finite differences. In this work, the gradient is defined as

$$
\nabla \mathrm{CO}_{2}=\frac{\Delta \mathrm{CO}_{2}}{\Delta \tau_{M}-\Delta \tau_{M+1}},
$$

where $\Delta \tau_{M+1}$ is the modeled differential optical depth computed using LBLRTM and an enhanced column $\mathrm{CO}_{2}$ value, which is the last estimated $\mathrm{CO}_{2}$ value plus a constant offset $\Delta \mathrm{CO}_{2}$. Currently, $\Delta \mathrm{CO}_{2}$ is set to a fixed $2 \mathrm{ppmv}$ enhancement over the previously estimated value. Finally, an updated estimate of the column concentration is computed based on

$$
\mathrm{CO}_{2(n+1)}=\mathrm{CO}_{2(n)}+\nabla \mathrm{CO}_{2}\left(\Delta \tau_{M}-\Delta \tau\right),
$$

where $\mathrm{CO}_{2(n)}$ represents the previously estimated column concentration value and $\mathrm{CO}_{2(n+1)}$ is the updated value. This iterative process is continued until the absolute difference, $\left|\Delta \tau_{M}-\Delta \tau\right|$, falls below the instrument noise threshold or the number of iterations exceeds a user-defined 
maximum. Nominally, this method converges in one to two iterations under a wide variety of atmospheric and environmental conditions.

The above-described retrieval algorithm has proven accurate for system deployments having relatively horizontal path lengths that are near or $<1 \mathrm{~km}$, as will be discussed in Secs. 3 and 4. In this case, $T / R H / P$ measurements from one or more in situ weather stations that are along or very near the observation lines of sight serve as a suitable approximation of atmospheric state in the retrieval process. However, expanding the system to measure path lengths up to $5 \mathrm{~km}$ that are often at a slant angle with respect to horizontal, presents new challenges in terms of accurately approximating atmospheric state along the observation lines of sight. First, the $T / R H / P$ may not be adequately represented by a single point measurement along a horizontal $5-\mathrm{km}$ path on the Earth's surface due to local gradients in $T / R H / P$ in latitude/longitude space. Second, a system having up to $5-\mathrm{km}$ path lengths that is deployed over a graded topographic landscape or in an urban environment would include transceiver and reflector hardware at heights that may differ by tens of meters above sea level from one another, resulting in slanted observational lines of sight. Again, $T / R H / P$ will likely not be adequately represented by a single point measurement along the line of sight due to vertical atmospheric gradients in $T / R H / P$. For these reasons, improvements have been made to the retrieval algorithm to provide higher fidelity retrievals for the case of slanted path lengths that are $>1 \mathrm{~km}$ in length.

To account for gradients in $T / R H / P$ along slanted path lengths $>1 \mathrm{~km}$, multiple weather stations that are within or near the intersecting chord grid are utilized to estimate $T / R H / P$. A given chord (observational line of sight) is divided into multiple segments $(\sim 1 \mathrm{~km}$ each). $T / R H / P$ is estimated for each segment using a distance weighted average of the surrounding weather stations. An additional adjustment is made to the estimated $P$ per chord segment using a lapse rate equation to account for the height above sea level of each chord segment. Finally, the $\mathrm{CO}_{2}$ concentration of each chord segment is retrieved and summed to give the final estimate of $\mathrm{CO}_{2}$ concentration along the full chord length. This segmented retrieval approach provides more accurate retrievals of $\mathrm{CO}_{2}$ for slanted path lengths $>1 \mathrm{~km}$.

As part of the GreenLITE design and development effort, a full set of simulation-based analyses were performed to quantify the impact of uncertainties in an atmospheric state, e.g., nearsurface temperature, water vapor concentrations and pressure, and instrument noise characteristics on retrieved $\mathrm{CO}_{2}$ concentrations. These analyses were modeled after those described in Ref. 21, and include an assessment of the impact of water vapor concentration on uncertainties in retrieved values. While uncertainties in both water vapor and temperature may significantly impact retrieval error characteristics depending on line selections, the chosen on/offline combination was found to have an error on the order of $0.2 \mathrm{ppmv}$ in retrieved $\mathrm{CO}_{2}$ concentrations given a $10 \%$ error in relative humidity. An additional source of error is the knowledge of the transmitted wavelengths. The current implementation of GreenLITE does not use locked lasers and slight drifts can cause measurement errors. Using precise thermal and current control, we have been able to maintain the GreenLITE wavelengths to $\pm 0.6 \mathrm{pm}$. Since the GreenLITE measurement is made at or near surface pressure, where the absorption line has maximum Doppler broadening, it is relatively insensitive to small wavelength changes when operating at the peak of the line for online and in the wings for the offline and is estimated to contribute up to a $0.5 \%$ ( 2 ppmv) error as implemented. This is in contrast to a space or airborne measurement where the vertical structure of the absorption cross-section would result in a much larger error. As we progress the measurement technology and strive for higher accuracy, it will likely become necessary to lock the lasers to an absorption feature or to continuously monitor the wavelength and use the measured values in the retrieval.

\subsubsection{Estimating two-dimensional fields of $\mathrm{CO}_{2}$ concentrations from tomographic samples}

The retrieved $\mathrm{CO}_{2}$ concentration values are then employed in an additional minimization scheme that constructs 2-D models of the underlying field concentrations. In traditional tomographic applications, the number of backprojections (chords) and angles should optimally approach or exceed the number of pixel elements in the resulting 2-D image, and often the scan geometry is optimized where possible to ensure high-fidelity, unconstrained reconstructions. In the 
GreenLITE case, the field is undersampled, and the scan pattern is limited due to the number of deployed transceivers and reflectors, site topography, and both natural and man-made barriers. While a number of approaches have been proposed and implemented for a variety of similar applications $\mathrm{s}^{22-24}$ to optimally constrain this type of problem, the current baseline method models the underlying field concentrations as the sum of a set of analytical functions that are designed to describe the underlying $\mathrm{CO}_{2}$ background distribution as well as a set of plume-like source terms. While a number of functional forms have been assessed as part of this work, the following analytical model describes the baseline 2-D field contained within the transceiver chord boundaries:

$$
F_{\mathrm{CO}_{2}}(x, y)=a+b x+c y+d x y+\sum_{n=0}^{N} \alpha_{n} e^{-\beta_{n} x_{r}^{2}} e^{-\gamma_{n} y_{r}^{2}}
$$

where $F(x, y)$ is the resulting $\mathrm{CO}_{2}$ concentration as a function of the $x / y$ location, and the linear model on the right side describes the field elements. The first four terms in Eq. (4) describe a basic background term that has a constant offset and is allowed to vary linearly as a function of $x$ and $y$. The variable $a$ describes the average background concentration, and the remaining elements describe a simple linear gradient across the field. The summation represents a set of simple Gaussian plumes to describe potential localized $\mathrm{CO}_{2}$ sources. Each 2-D Gaussian is a function of a normalized set of $x_{r}$ and $y_{r}$ parameters defined as

$$
x_{r}=\sqrt{\left(x-x_{n}\right)+\left(y-y_{n}\right)} \cos \left(\theta_{n}\right),
$$

and

$$
y_{r}=\sqrt{\left(x-x_{n}\right)+\left(y-y_{n}\right)} \sin \left(\theta_{n}\right),
$$

where $\left(x_{n}, y_{n}\right)$ is the plume center and $\theta_{n}$ is the angle of rotation. The terms $\alpha_{n}, \beta_{n}, \gamma_{n}$ define the maximum concentration and $x / y$ widths of each plume. The number of chords limits the number of supportable modeled plumes. The upper limit is defined as the number of chords minus the four background parameters, divided by the number of parameters. In this case, each plume is defined by six parameters $\alpha_{n}, \beta_{n}, \gamma_{n}, x_{n}, y_{n}$, and $\theta_{n}$. For example, a site with 42 intersecting chords limits the number of plumes to a maximum of $6[(42-4) / 6=6.3]$. In practice, the number of supportable plumes is on the order of 3 to 4 due to the ability of the minimization technique to uniquely assign values to each element. $F(x, y)$ is constructed using an optimization scheme that minimizes the difference between retrieved $\mathrm{CO}_{2}$ ppmv values and modeled values constructed through discrete integration of $F(x, y)$. This is achieved by minimizing the sum of the root-mean-square error differences between these values using a sequential least squares programming (SLSQP) optimization algorithm. SLSQP provides a standardized framework for minimizing a user-defined cost function given a set of additional parametric constraints. In this instance, the cost function was defined as the sum of the squared differences between a set of observed chord values and modeled chord values created by averaging equally spaced samples along a given chord set for the model described in Eq. (4). An additional weighting function was also placed on this overall metric so that the ratio of $\beta_{n}$ to $\gamma_{n}$ did not exceed some maximum, ensuring that plumes, irrespective of direction, had some finite width. As part of the overall minimization scheme, the model parameters were constrained (1) to ensure that the plumes were positive in nature and represented source and not sink terms, (2) to constrain plume source locations to the enclosed sampling space, and (3) to restrict plume source amplitudes to within physically realizable bounds for $\mathrm{CO}_{2}$ emissions.

Figure 4 shows the results from a typical reconstruction based on synthetic data. In this example, Fig. 4(a) shows a typical synthetic field containing two centrally located Gaussian plumes on a constant background. The red lines indicate the chords associated with a typical two-transceiver configuration with 10 reflectors on each of three sides. Next, the average concentration for each chord is computed based on discrete samples along each chord length. Figure 4(b) shows a simple backprojection of the synthetic chord samples. Each dot in the resulting 2-D plot denotes the average value of all chord values that intersect the point of interest. While the resulting sparse backprojection provides little information about the true distribution, it does provide a crude first guess at potential plume locations. Finally, Fig. 4(c) describes the 
(a)

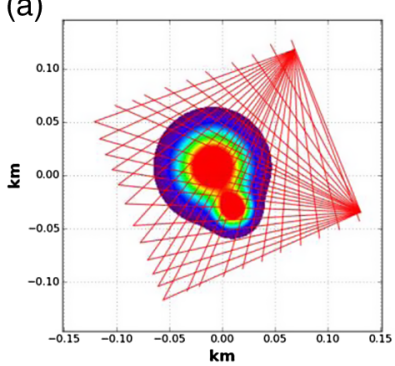

(b)

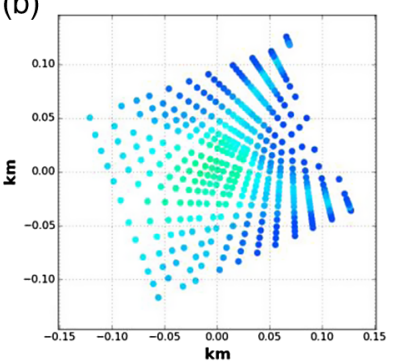

(c)

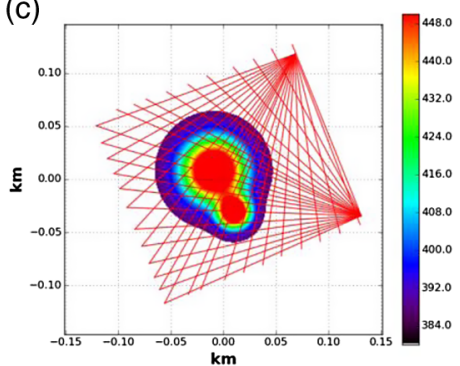

Fig. 4 Simulated 2-D reconstruction. (a) A synthetic scene and simulated chords for a typical twotransceiver configuration. (b) A simple backprojection of the synthetic chord samples constructed from the field (a), and (c) the resulting reconstruction.

resulting reconstruction based on the synthetic data derived from the Fig. 4(a) and the reconstruction algorithm described earlier. This tomographic reconstruction method provides a robust mechanism for estimating well-defined plume-like sources.

\subsubsection{Data processing and data dissemination}

GreenLITE utilizes an integrated cloud-based data collection, data processing, and data dissemination approach. At the center of this design is a common database implementation based on open-source software, MySQL, and a python web framework, hosted at Amazon Web Services (AWS). The core database provides common queryable secure storage and enables deployment of standard mechanisms for data ingest, data access, and instrument and site monitoring, as well as data dissemination for real-time analysis. Raw instrument data, instrument metadata, and in situ measurements from each transceiver, as well as data from colocated weather stations, are transferred to the cloud-based applications via cellular data services using secure web-based protocols. Portions of these data are distributed in autonomous fashion to downstream processing elements, which are hosted in a distributed fashion and compute the column $\mathrm{CO}_{2}$ concentration values and 2-D distributions described above. The results are also stored and indexed in the same centralized database implementation. Finally, access to instrument, in situ, and processed data are provided to end users and applications via common web interfaces. An example of the end-user oriented web interface is shown in Fig. 5.

This web interface is configured in an automated fashion based on site-specific information, e.g., transceiver geographic location and sample times, and provides both user-oriented real-time dynamic site-specific decision support information, as well as global remote access

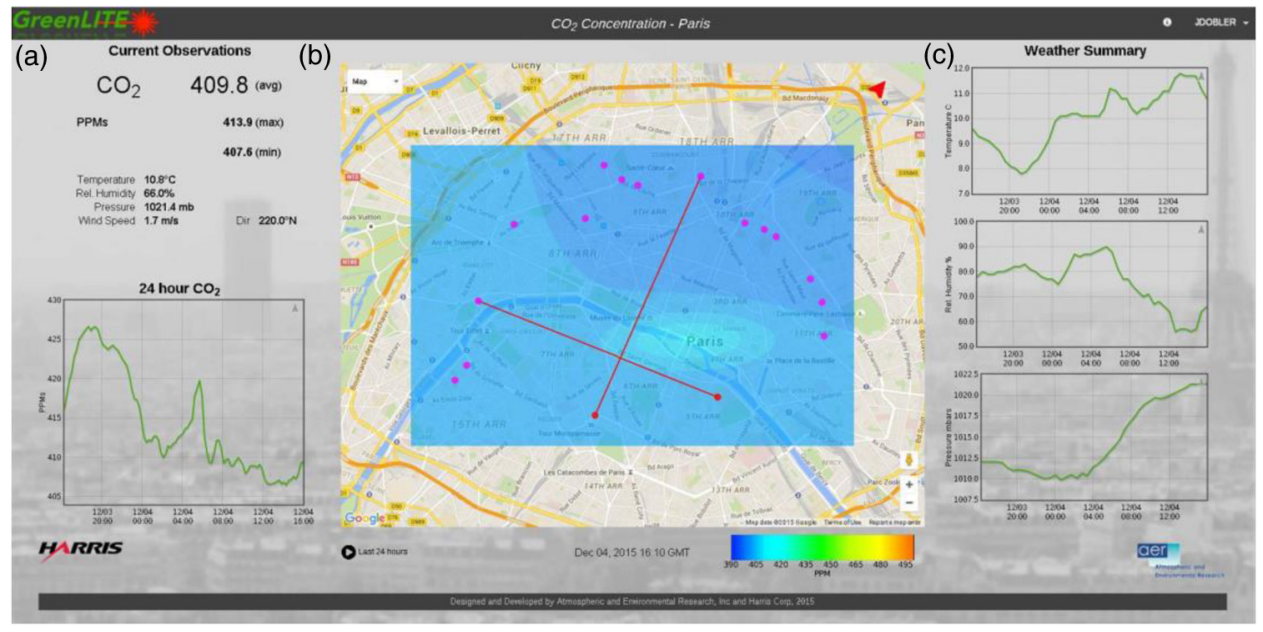

Fig. 5 Examples of the user interface: current average $\mathrm{CO}_{2}$ concentration and average concentration as (a) a function of time, (b) latest 2-D reconstruction, and (c) weather data. 
(a)

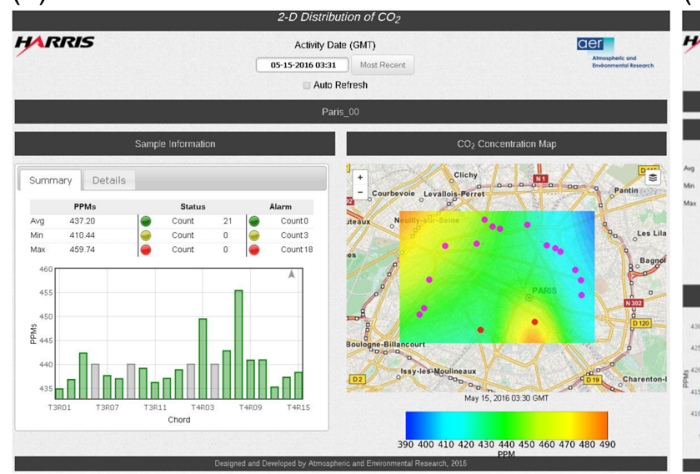

(b)

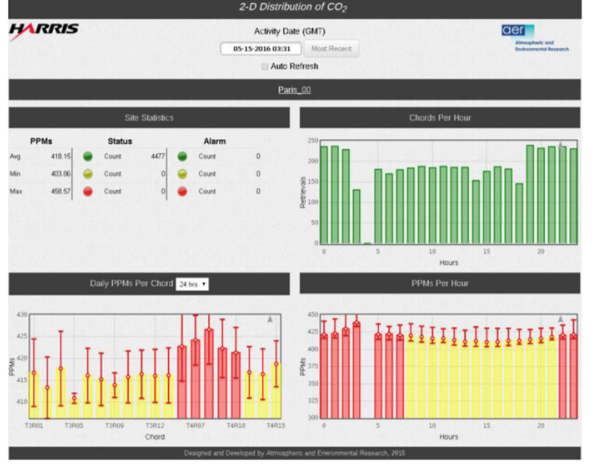

Fig. 6 Examples of next-level information available in web interface: (a) latest chord data and 2-D reconstruction, and (b) summary data showing chord statistics and trends over most recent 24-h period.

to up-to-the-minute instrument monitoring, quality control, system statistics, and ancillary information. This system provides top-level summary information that includes current integrated concentration values on a chord-by-chord basis, georeferenced 2-D reconstructions, and indicators of anomalous conditions based on user-defined thresholds. In addition, the same interface provides user-selectable time-series-based displays and analyses of retrieved as well as raw sample information and metadata. Finally, it provides real-time displays of ancillary information from instrument health and safety sensors, colocated weather stations, and webcams. Some of these functions are illustrated in the displays shown in Fig. 6.

Top-level information is available to authorized users through the main page shown in Fig. 5. The database structure allows individualized access to additional measurement details through the other next-level webpages like those shown in Fig. 6. The system administrator can provide access to different levels on a per user basis as required.

Additional information is also available through the web interface such as: 2-D reconstruction time series, instrument telemetry, raw signal and differential transmission data, and server administration details, each accessible in near-real-time or historic views. The web interface is highly customizable for application-specific needs.

\section{Field Deployments and Objectives}

The GreenLITE system has undergone several validation efforts and field deployments to demonstrate its capabilities. The initial GCS system with an optimal chord range of $1 \mathrm{~km}$ was first tested at a local test facility near Fort Wayne, Indiana, and then tested over a 3-week period in July to August of 2014 at the ZERT test facility operated by Montana State University in Bozeman, Montana. The system was then deployed at an operational GCS site in Illinois for an extended period of more than 6 months from February to August of 2015. The full $5-\mathrm{km}$ path length of the second instrument, designed and developed in 2015, was first tested at its intended 5-km path length at the National Oceanic and Atmospheric Administration (NOAA) Boulder Atmospheric Observatory (BAO) tower site and, at the time of this writing, is operating in Paris, France, as part of an international collaboration. This section provides a description of each of these test sites and the objectives for each. Section 4 provides information about the tests conducted and reviews relevant results to date for each site.

\subsection{Harris Farm Test Site}

The Harris farm test site is located in New Haven, Indiana, and serves as the testbed for a number of active and passive instruments under development at the Harris Fort Wayne campus. The site has a research trailer located to allow a clear view over an agricultural field of $\sim 700 \times 500 \mathrm{~m}$, as shown in Fig. 7. The site is equipped with a full-time Davis Weather Vantage Pro station for 
(a)

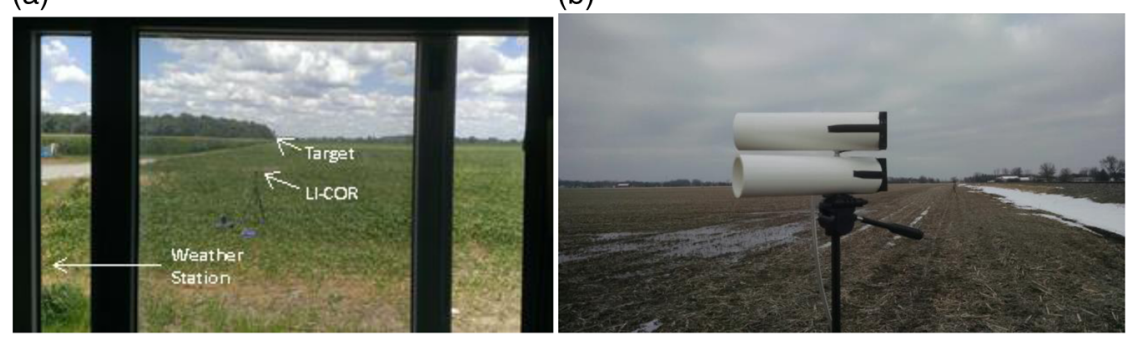

Fig. 7 (a) View from Harris farm test site trailer window, and (b) simulated plume setup to test 2-D reconstructions by subjecting specific chords to $\mathrm{CO}_{2}$ enhancement.

meteorological information and has a target located $680 \mathrm{~m}$ from the trailer that can be used for various tests with different reflector surface materials. For GreenLITE, component level testing was done in the laboratory at our facility in Fort Wayne, while the initial system level transceiver testing was done in the trailer and primarily consisted of verifying instrument performance parameters, such as signal-to-noise ratio (SNR), pointing, divergence angles, etc. Following completion of the second transceiver, the farm test site was used to do side-by-side comparisons between the two transceivers for a number of retroreflector configurations. Additionally, this site provided a location to test various configurations of the full GreenLITE system prior to deployment to the ZERT site and the Illinois Basin-Decatur Project (IBDP) site, discussed in the following sections. The primary objectives for testing at this site were to verify that the transceivers met design specifications, demonstrate consistent calibration between the two transceivers, and evaluate potential drift. The site also enabled the first tests of the algorithms to generate the 2-D concentration estimate maps and the interfaces between the hardware and the AWS database, retrievals, and data dissemination aspects of GreenLITE. The original GreenLITE system was set up over an area of $\sim 0.2 \mathrm{~km}^{2}$ with two transceivers and 30 retroreflectors. Open-ended tubes were placed immediately in front of selected retroreflectors, and $\mathrm{CO}_{2}$ was injected into the tubes to increase the column-averaged concentration of those specific chords. From the point of view of the GreenLITE system, this was equivalent to the release of a $\mathrm{CO}_{2}$ plume at the location where the selected chords intersected. The test setup is shown in Fig. 7. Furthermore, the farm site provided the first test of harsh environment autonomous operation tests in December 2014 to January 2015. Initial testing for the 5-km system was also done at the farm, but the available range was not adequate to fully test the system.

\subsection{Zero Emissions Research and Technology Field Site}

The original GreenLITE system was set up at the ZERT site in Bozeman, Montana, with the goal of demonstrating the ability to deploy the system remotely and to demonstrate its ability to detect controlled $\mathrm{CO}_{2}$ releases from an underground pipeline. The ZERT site, operated by Montana State University, is equipped with a $70-\mathrm{m}$ pipe buried $\sim 2 \mathrm{~m}$ below the surface from which they are permitted to release $\mathrm{CO}_{2}$ at a rate of up to 0.3 ton/day. ${ }^{8}$ The site was designed to test monitoring, reporting, and verification approaches and instrumentation, with the release rate from the 70-m underground pipe having been scaled to the required sensitivity needed for ensuring $99 \%$ containment of a full-scale GCS site. ${ }^{25}$ The GreenLITE system was operated at the site over a 4week period in fall 2014 and demonstrated the ability to detect not only the controlled release but also the $\mathrm{CO}_{2}$ signature from a manure pile located near the test site. The typical ZERT transceiver deployment, an extended aerial view of the ZERT site, and a graphical depiction of the transceiver and retroreflectors for the ZERT deployment are shown in Fig. 8.

\subsection{Illinois Basin-Decatur Project}

The first GreenLITE extended deployment was to a GCS site in Illinois. The primary objective of this deployment was to verify that the system could be operated autonomously on an active GCS site over an extended period of time and wide range of operating conditions. The system was set 

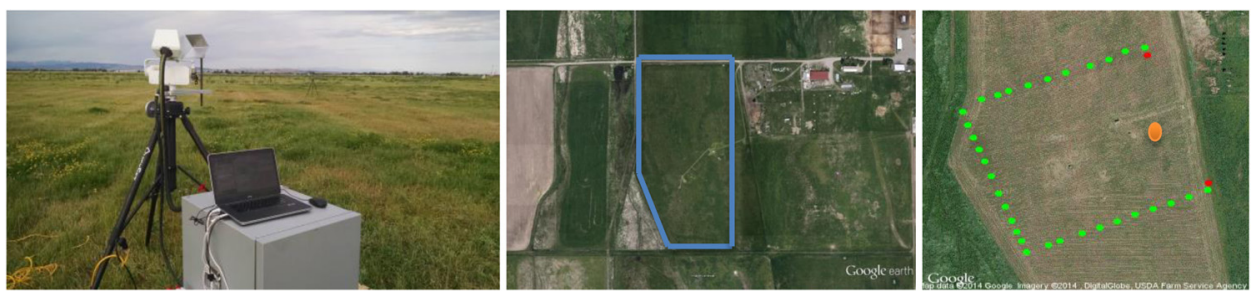

Fig. 8 Initial GreenLITE system testing at the ZERT facility in Bozeman, Montana. Orange dot indicates where the in situ instrument was placed for the comparisons in Sec. 4.

(a)

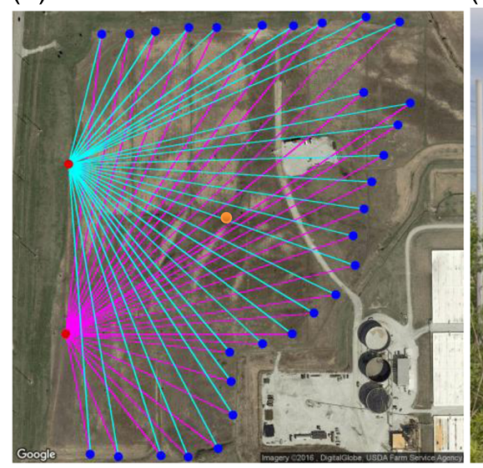

(b)

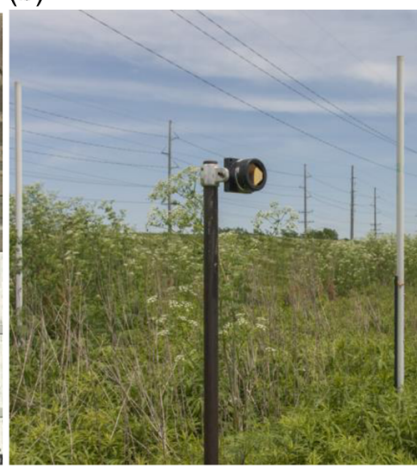

(c)

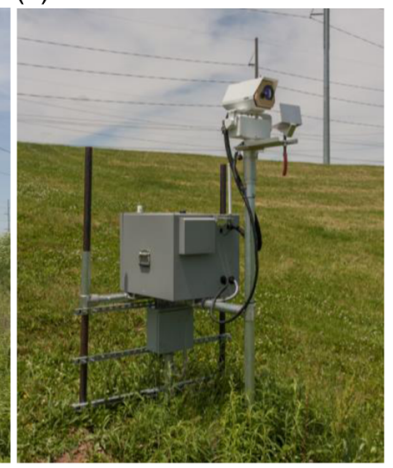

Fig. 9 Deployed system at the IBDP in Decatur, Illinois. (a) Site layout, (b) mounted reflector, and (c) installed transceiver. Orange dot in (a) indicates approximate location of the LI-COR Biosciences ${ }^{\circledR}$-based multiplexer.

up to cover a heavily vegetated, irregularly shaped area of $\sim 0.2 \mathrm{~km}^{2}$. The GreenLITE system was operated for $>4000 \mathrm{~h}$ in temperatures of $-15^{\circ} \mathrm{C}$ to $+35^{\circ} \mathrm{C}$ with minimal human interaction required. The IBDP site configuration and typical retroreflector and transceiver deployment are shown in Fig. 9.

\subsection{Boulder Test Site}

The extended-range GreenLITE system was sent to Boulder, Colorado, in August 2015, with the objective of demonstrating both the longer-range capabilities of the transceivers and the segmented retrievals for slant paths as described in Sec. 2.2.1. This site also allowed for a well-characterized and calibrated in situ system to provide some estimate of the accuracy of the extended-range GreenLITE. It was tested over a 3-week period adjacent to the NOAA BAO tower near Boulder. The BAO tower is a $300-\mathrm{m}$ tower instrumented with a highly calibrated LI-COR-based measurement system. The LI-COR system samples at three ports, located at 22, 100 , and $300 \mathrm{~m}$ AGL, and then follows this sampling with the sampling of a test gas traceable to WMO standards. In addition, the tower provides high accuracy meteorological data for calibration and validation of our portable weather stations. The transceivers were colocated first at $2 \mathrm{~km}$ and then at $5 \mathrm{~km}$ from the tower, and retroreflectors were mounted at five heights on the tower. These test configurations allowed side-by-side comparisons between the transceivers, as well as the opportunity to compare retrieved concentrations to the measurements made by the tower instrumentation. Figure 10 shows an aerial view of the tower (right yellow place-marker) and the 2 and $5-\mathrm{km}$ transceiver locations (central and left yellow place-markers) as well as a typical transceiver and retroreflector tower mount.

\subsection{Paris, France}

The extended-range GreenLITE system was deployed over a $25-\mathrm{km}^{2}$ area of central Paris, France, in November 2015. The deployed system consists of two transceivers and 15 


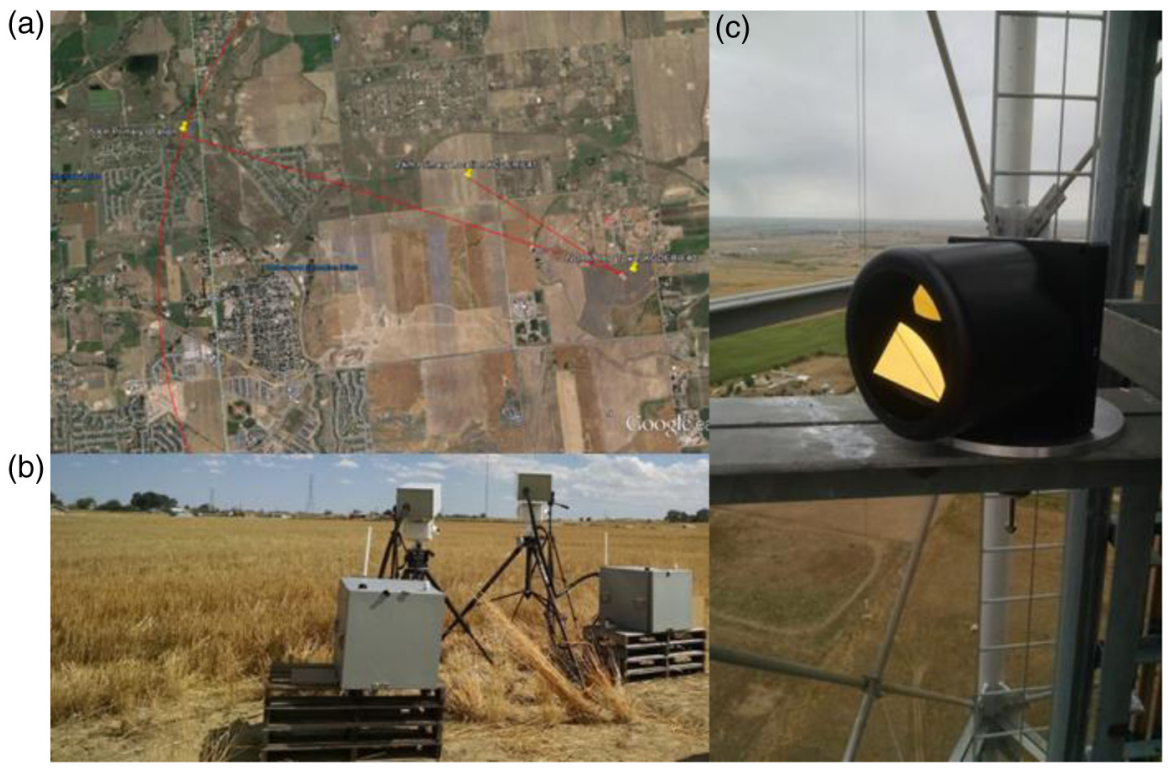

Fig. 10 Test setup for 5-km GreenLITE system at the BAO near Boulder, Colorado. (a) Site layout for 2- and 5-km tests, (b) side-by-side testing of the transceivers, and (c) a mounted reflector on the $\mathrm{BAO}$ tower.

retroreflectors placed on top of buildings roughly arranged in a hemisphere as shown in Fig. 11(c). The availability of buildings of suitable height and with lines of sight to the transceivers limited the number of retroreflectors. The objectives for the deployment to Paris were to demonstrate the system in an urban environment, show feasibility of deploying a system in an urban setting, and evaluate the ability of these data to provide additional information and constraints at scales relevant to transport models over a large urban area, which is challenging with the present in situ measurements. The system has been operated continuously since deployment and is scheduled to remain in operation through the end of 2016. Parallel to the GreenLITE system, the Laboratoire des Sciences du Climat et de L'Environnement (LSCE) continuously operates multiple sites throughout the Paris metropolitan area as part of their urban GHG monitoring system (e.g., Refs. 2 and 26). The two closest sites are the continuous station in the Cite des Sciences et de l'Industrie (CDS) and an instrument deployed at Jussieu during a field campaign in March 2016. The CDS site is located in a park in the northeast of central Paris $\left(48.896^{\circ}\right.$ $\mathrm{N}, 2.388^{\circ} \mathrm{E}$ ) and equipped with a cavity ring-down spectrometer analyzer (G2401/Picarro). The air is sampled on the roof of the building at $34 \mathrm{~m}$ AGL. Variations in $\mathrm{CO}_{2}$ concentrations are very large, ranging from 390 to 550 ppmv (hourly means), which can be detected easily with the G2401 due to a typical repeatability of $0.02 \mathrm{ppmv}$ for this instrument. The instrument is

(a)

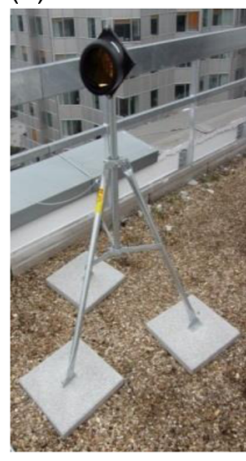

(b)

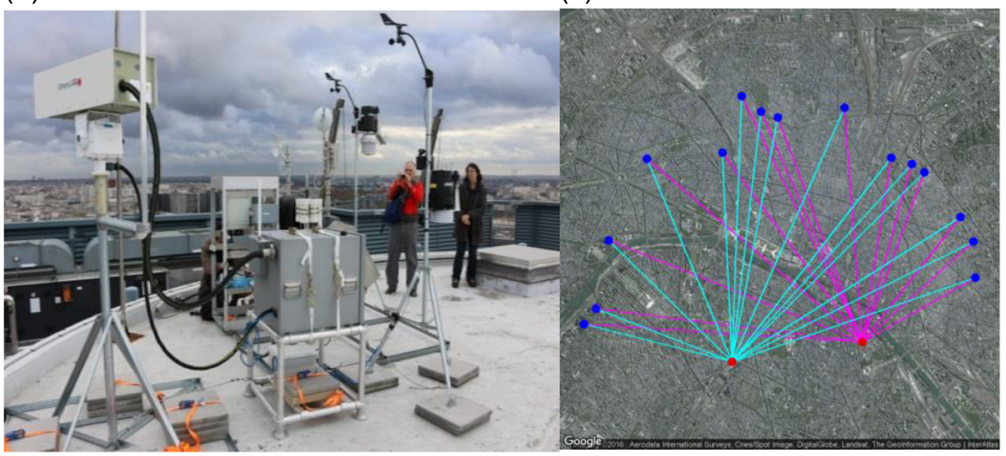

Fig. 11 Deployed system in Paris, France. (a) Typical reflector installation, (b) transceiver installation, and (c) overall layout. 
calibrated with three reference gases every 6 months and with one reference gas twice per day. All reference gases are calibrated at LSCE with a suite of six NOAA/WMO cylinders (WMOCO2-X2007 scale). The raw data are transferred once per day and processed at LSCE. For the colocated field campaign in Jussieu, a Thermo Fisher Delta Ray isotope ratio IR spectrometer was installed at the QUALAIR Laboratory adjacent to the Jussieu tower at a height of $122 \mathrm{~m}$ above sea level (25-m AGL). It was deployed with two pure $\mathrm{CO}_{2}$ standards for carbon isotopes and a concentration reference gas. Before deployment, the typical performance of the Delta Ray was assessed using long-term target measurements and revealed a reproducibility of better than $0.1 \mathrm{ppmv}$.

\section{Analysis and Results}

The GreenLITE system deployments described in Sec. 3 have produced a significant amount of test and validation data over the past several years, including observations from the ZERT test facility in Bozeman, Montana, a GCS site in Decatur, Illinois, as part of a field campaign in Boulder, Colorado, and an ongoing installation in Paris, France. Each of these deployments exercised and validated various aspects of the GreenLITE system hardware, data collection system, retrieval algorithms, and 2-D reconstruction algorithms. The resulting observations include both an extensive set of retrieved $\mathrm{CO}_{2}$ concentration values for a number of geographically diverse locations and environmentally significant conditions as well as the corresponding 2$D$ reconstructions. As part of these efforts, additional data have been obtained to cross-validate the resulting data products, and a number of field experiments have been undertaken to quantify the performance of the 2-D reconstruction algorithm. A summary of these field experiments and the comparison of the resulting observations to in situ measurements are given in the sections below.

\subsection{Retrieving $\mathrm{CO}_{2}$ Column Amounts}

For all of the field tests/deployments except the farm test site-ZERT, IBDP, Boulder, and Paris-comparisons could be made of the $\mathrm{CO}_{2}$ concentration values retrieved with the GreenLITE system to measurements from independent in situ instrumentation with varying degrees of calibration. In all cases, the independent concentrations were point sources measurements. Since GreenLITE measures the $\mathrm{CO}_{2}$ concentration through an integrated column of air, absolute comparisons between GreenLITE concentrations and those from the independent point source measurements are not expected to equate but should track each other. However, quantitative comparisons are made to show that (1) the GreenLITE system is measuring and computing realistic $\mathrm{CO}_{2}$ concentrations that are representative of the actual present $\mathrm{CO}_{2}$ levels and (2) the GreenLITE system is able to track local changes in $\mathrm{CO}_{2}$ concentrations over both short(minutes) and long- (months) time scales.

Figure 12 shows a representative set of colocated in situ point measurements collected with a LI-COR-based West Systems Chamber that was placed in a stationary location within the chord measurement grid during the ZERT field test, and the corresponding $\mathrm{CO}_{2}$ chord values retrieved with the GreenLITE system. The averaged retrieved values track in situ measurements over an $\sim 18$-h period. The plot demonstrates not only that average GreenLITE and in situ measurements agree well but also that the GreenLITE system has tracked short-term fluctuations in local $\mathrm{CO}_{2}$ concentration levels.

The plots in Fig. 13 show comparisons to an LI-COR-based multiplexer operated by the Illinois State Geological Survey at the IBDP site. The in situ height shown in the figure was $2.44 \mathrm{~m}$ off the ground while the GreenLITE measurement included four nearby chords ranging from about 2-m off the ground to $3.5-\mathrm{m}$ off the ground at this location. The LI-COR instrument provides a point source measurement, which is quite different than the integrated path measurements of the GreenLITE system averaged over multiple lines of sight. Therefore, direct comparisons between the two would not necessarily provide a good quantification of measurement accuracy. Rather, one would expect the relative trends of measured concentration to track between the two. In fact, general trends are tracked extremely well, as seen in Fig. 13(a). 
(a)

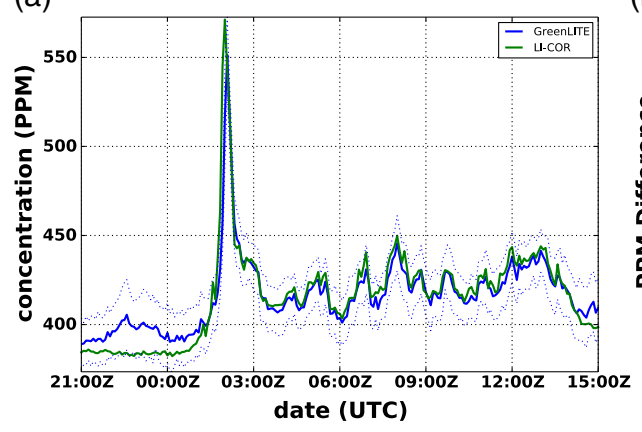

(b)

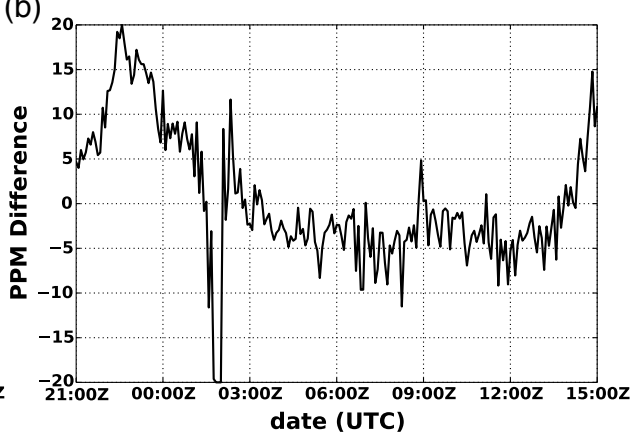

Fig. 12 Representative comparison of GreenLITE retrieved column $\mathrm{CO}_{2}$ values (blue) and colocated in situ field measurements (green) during the ZERT field test. (a) Dotted blue lines denote the 5-min max and mins of all chords. (b) average differences on a 5-min basis.
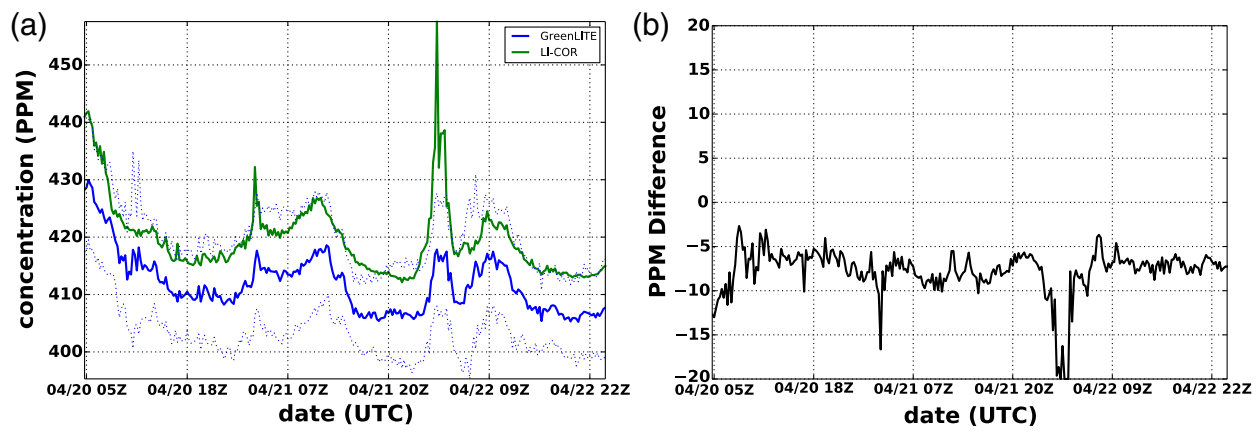

Fig. 13 Representative comparison of GreenLITE retrieved column $\mathrm{CO}_{2}$ values (blue) and colocated in situ field measurements (green) during the IBDP test. (a) Dotted blue lines denote the 1-h average max and min values for all chords. (b) Average differences on a 5-min basis.

The integrated path measurements typically read lower than the in situ instrument by 5 to 10 ppmv, as seen in Fig. 13(b). Like the ZERT site LI-COR, the LI-COR system used at IBDP does not regularly sample calibration gas; thus, the absolute calibration is unknown and cannot be used as an absolute reference by which to evaluate GreenLITE accuracy. However, it still provided relative measurements to support our assertion that GreenLITE's observed trends are real and due to changes in $\mathrm{CO}_{2}$.

During the 5-km GreenLITE testing, a portion of the Boulder field test included two transceivers placed $5 \mathrm{~km}$ from the $\mathrm{BAO}$ tower $\sim 1 \mathrm{~m}$ above ground and two retroreflectors placed on the tower $\sim 180$ and $120 \mathrm{~m}$ (respectively) above ground. A subset of retrieved $\mathrm{CO}_{2}$ concentration values for all four measurement paths (two transceivers $\times$ two retroreflectors) is shown in Fig. 14 (blue) along with independent measurements from a LI-COR-based instrument operated by the NOAA Earth Systems Research Laboratory on the BAO tower that samples sequentially at 22, 100 , and $300 \mathrm{~m}$ above ground (green). The plot spans $3 \mathrm{~h}$ on 10 September 2015. In addition to the LI-COR-based tower data, a Picarro instrument operated by the University of Colorado was located $\sim 3 \mathrm{~m}$ above the surface near the base of the tower; it is also shown in Fig. 14 (red). Again, note that the LI-COR and Picarro measurements represent points in space, while GreenLITE measured integrated paths. Even so, a relatively small average bias of 2.64 ppmv between the GreenLITE and the tower LI-COR measurements was calculated for the time period shown. To better observe and compare concentration trends among the three measurements types, the GreenLITE data are corrected by $2.64 \mathrm{ppmv}$ in Fig. 14(b) to minimize differences in the well-mixed portion, and, once again, the measurements track well.

Retrieved $\mathrm{CO}_{2}$ concentrations from the ongoing system deployment in Paris have been compared with independent Picarro measurements from the CDS site just northeast of the GreenLITE foot print, as shown in Fig. 15. The plots span 20 days in January 2016 and 

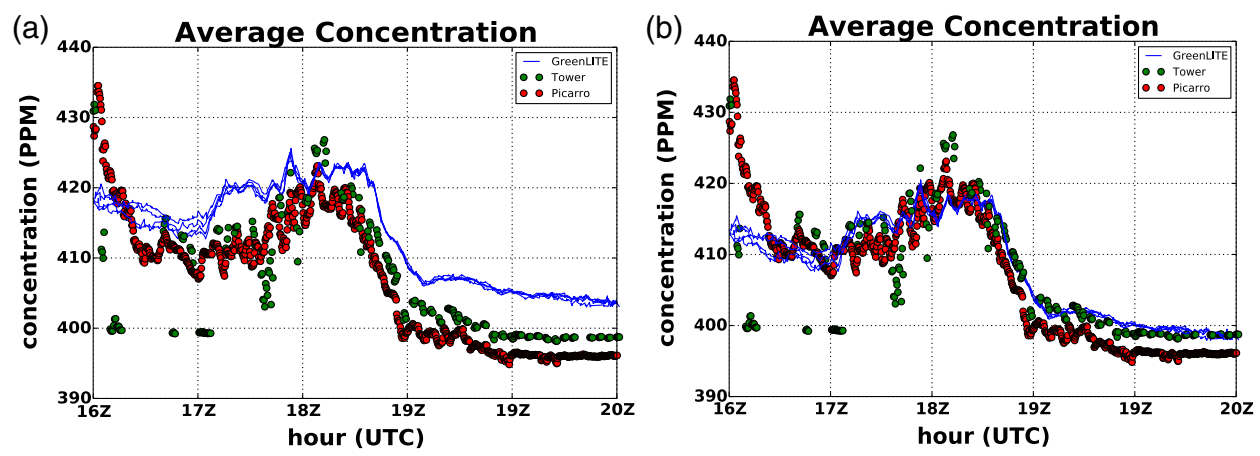

Fig. 14 Comparison of averaged GreenLITE retrieved column $\mathrm{CO}_{2}$ values (blue), colocated in situ averaged LI-COR (green), and Picarro (purple) field measurements during Boulder field testing. A 2.64-ppmv bias correction is applied to GreenLITE data in (b).

demonstrate the GreenLITE system's ability to track changes in local $\mathrm{CO}_{2}$ concentration over several days in an urban environment.

Figure 16 shows comparisons to the Thermo Fisher Delta Ray located at the Pierre and Marie Curie University, over a span of about 2 weeks of March 2016. The comparisons with both the Picarro and the Thermo Fisher in situ devices show very good correlation with the average GreenLITE data, although absolute values vary at times partly since they are fundamentally not measuring the same air mass due to the remote nature of the GreenLITE measurement, and some differences are expected. The differences in air mass assumption are also supported by the comparisons between the two in situ instruments shown in Fig. 17, which vary from each other significantly due to different locations, despite being regularly calibrated using WMO traceable gases from the same source, with verified high accuracy.

Assessing GreenLITE measurement bias is a challenging endeavor as there is no independent measure of "truth" over the same atmospheric volume observed by the system. Figure 17 shows

(a)
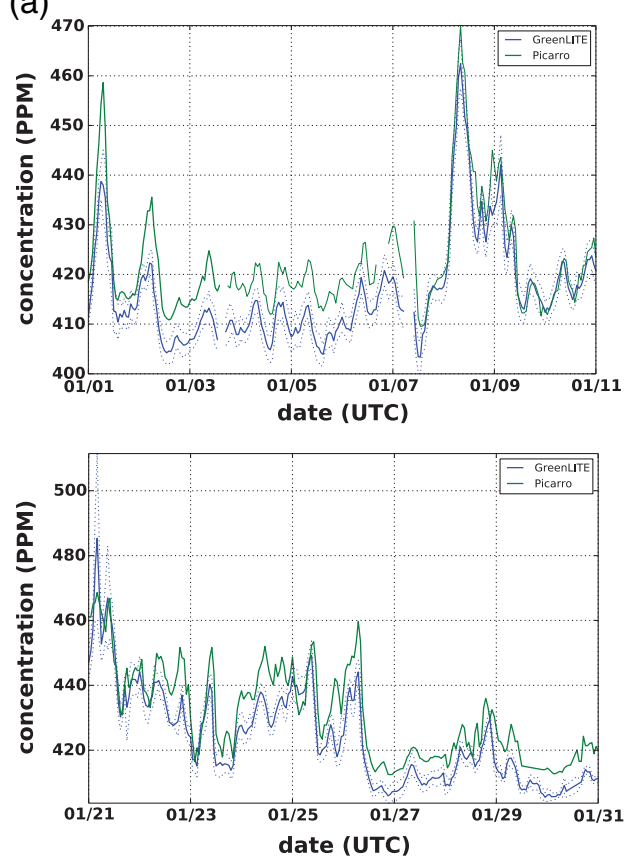

(b)
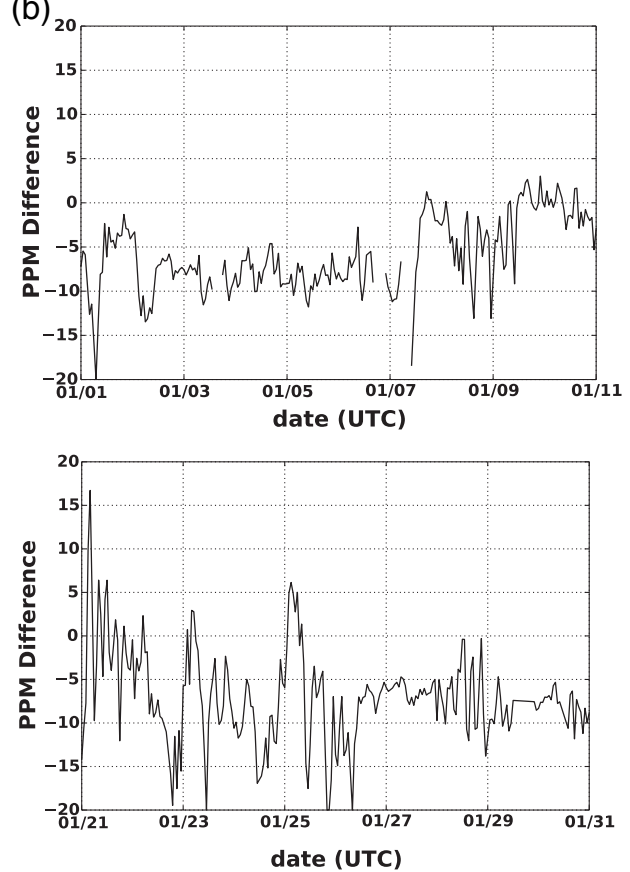

Fig. 15 (a) Comparison of GreenLITE retrieved hourly average column $\mathrm{CO}_{2}$ values (black) and colocated in situ field measurements (blue) from operational Paris deployment. Dotted blue lines denote the $1 \mathrm{~h}$ average max and min values for all chords. (b) Residual difference between GreenLITE retrieved and in situ measured $\mathrm{CO}_{2}$ concentrations. 

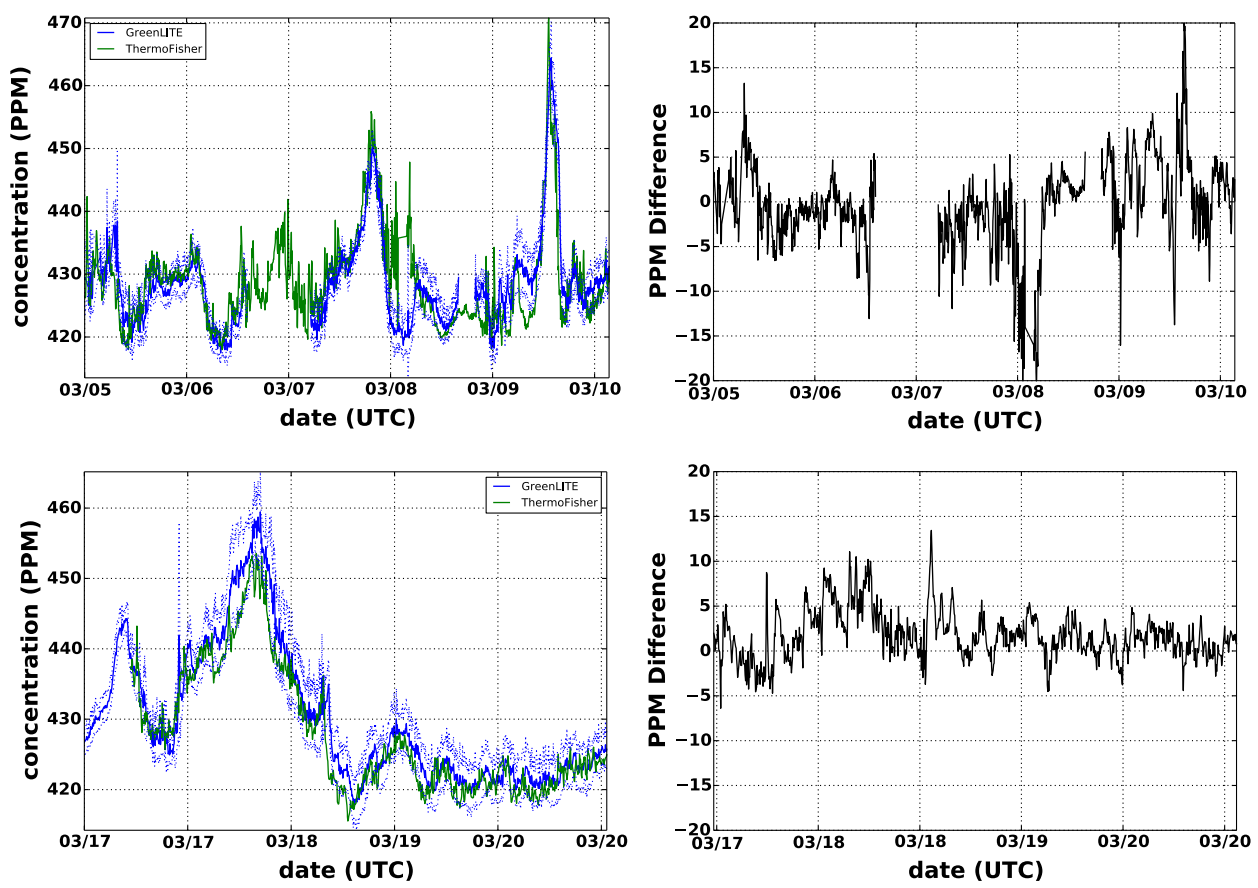

Fig. 16 Comparisons with the LSCE Thermo Fisher Delta Ray instrument located near the Jussieu tower but on a building $\sim 60 \mathrm{~m}$ lower than the transceiver on top of the Jussieu tower. Dotted blue lines denote the 5-min max and mins of all chords.

the differences between GreenLITE measurements and high accuracy in situ instruments, which are regularly calibrated using NOAA traceable gases, located near or within the GreenLITE field of view. This figure also shows the differences between in situ measurements within the same field of regard and illustrates the challenges faced in cross-calibration of independent measurements within a complex urban environment. The limited comparison with the Delta Ray, which was in close proximity to one of the GreenLITE transceivers, shows a very small difference

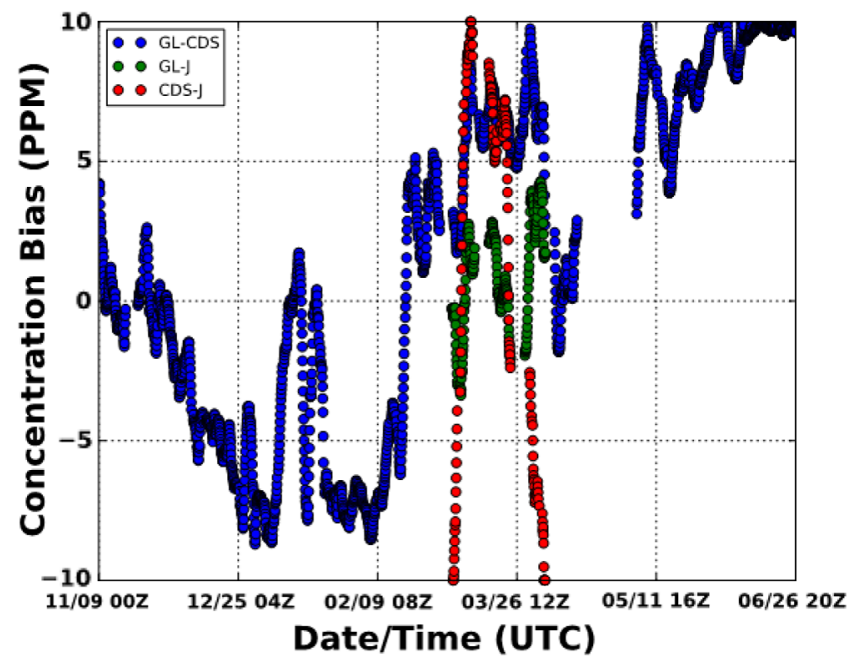

Fig. 17 Average differences between near-surface measurements of $\mathrm{CO}_{2}$ from three independent instruments, the Picarro at CDS (CDS), the Thermo Fisher Delta Ray near the Jussieu tower (J), and GreenLITE (GL). The plot shows the 4-h average differences between each; GreenLITE minus the Picarro, GreenLITE minus the Thermo Fisher Delta Ray, and the Picarro minus the Thermo Fisher Delta Ray. The Delta Ray data were only available for a limited period from 3 April to 4 July 2016, but they showed the smallest relative difference relative to the GreenLITE sensor. 
between the two. Temporal differences between the Picarro instrument at CDS and GreenLITE may be compounded by nonoverlapping foot prints and the fact that the CDS site is located in an urban park, which may enhance the observed differences as a function of season. The difference in location and height of the two in situ instruments is also likely a driver of the variability in the comparisons seen between the two.

Determining the precision of GreenLITE measurements from existing Paris data is also a challenge as it is not possible to map fluctuations within the 5-km chord measurements at a single point. Based on our airborne instrument ${ }^{13,14,15}$ and prior testing with the prototype GreenLITE system, we believe the inherent measurement precision is much better than 1 ppmv over the 5-km range, but it will have some additional variability driven by reduced chord length, alignment, wind, scintillation, and other factors that still need further evaluation to quantify their impacts. Figure 18 shows theoretical analysis results of the instrument measurement precision as a function of SNR and chord length (differential optical depth) based on $d \tau / \tau=400 /(\tau \times \mathrm{SNR})$. Evaluation of the GreenLITE measured absorption ratio using $10 \mathrm{~s}$ averages in a rural environment showed SNR values typically $>3000$ in the differential absorption ratio. For the original $1-\mathrm{km}$ system, measured values over a rural $1400 \mathrm{~m}$ path were $>4000$, which is equivalent to $<1 \mathrm{ppmv}$ precision and includes atmospheric variability. For the $5-\mathrm{km}$ system, initial testing at the BAO tower, also rural, showed SNR's over the 5-km path of $>3000$, which indicates a precision on the order of 0.25 ppmv. The BAO test data showed a potential bias of $\sim 2.6$ ppmv during the well-mixed time of day, but it was of very limited extent and more extended testing is required to properly evaluate the accuracy. Data from the system while deployed in Paris, that appear to have been from a relatively well-mixed time period in March ( $\sim$ months into the campaign) were randomly selected, and GreenLITE was found to have an SNR of 412 for a 3.4-km path and 423 for a $4.7-\mathrm{km}$ path (one path from each transceiver), which would indicate a precision of 2.3 and $1.57 \mathrm{ppmv}$, respectively, over a period from 9 a.m. to 12 p.m. For the Paris data, it is not possible to separate how much of the SNR reduction in the ratio is due to real variability in the urban environment versus instrument performance. Another metric of performance for the instrument is the stability of the calibration values, which showed SNR's $>10,000$ throughout the 1 year Paris experiment, which is equivalent to $0.06 \mathrm{ppmv}$ for the $5-\mathrm{km}$ case and $0.4 \mathrm{ppmv}$ for a $1-\mathrm{km}$ case, demonstrating the high stability of the instrument. At this time, we do not have adequate information to determine absolute accuracy of the system with confidence, but we have demonstrated it is very precise.

Finally, Fig. 19 shows hourly averaged $\mathrm{CO}_{2}$ concentration values from the GreenLITE system in Paris for a 40-h time span and demonstrates the GreenLITE system's ability to track expected diurnal $\mathrm{CO}_{2}$ patterns on an hourly basis.

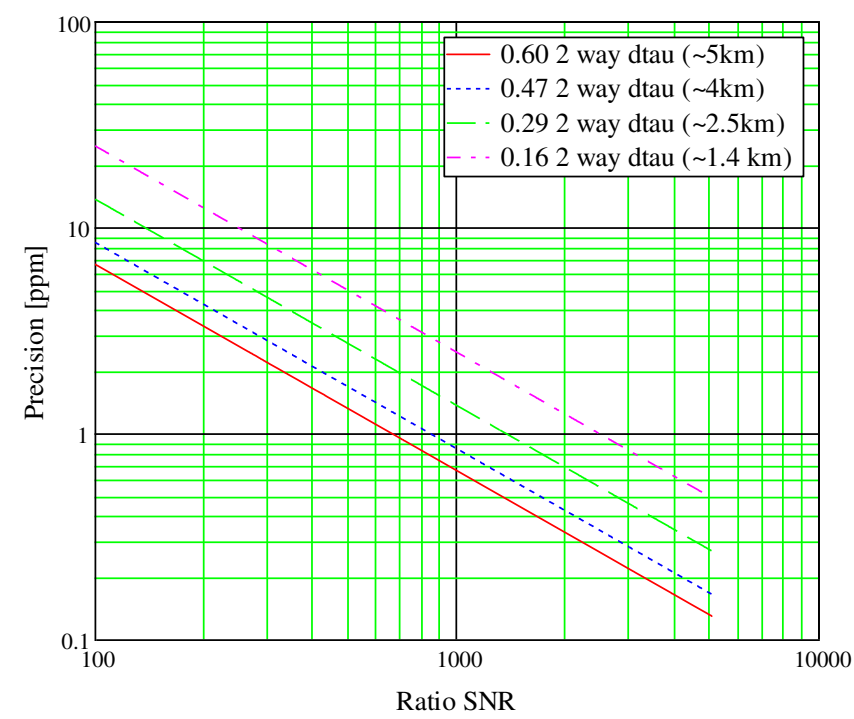

Fig. 18 System measurement precision versus instrument measured differential absorption ratio SNR for a set of differential optical depths (path lengths). 


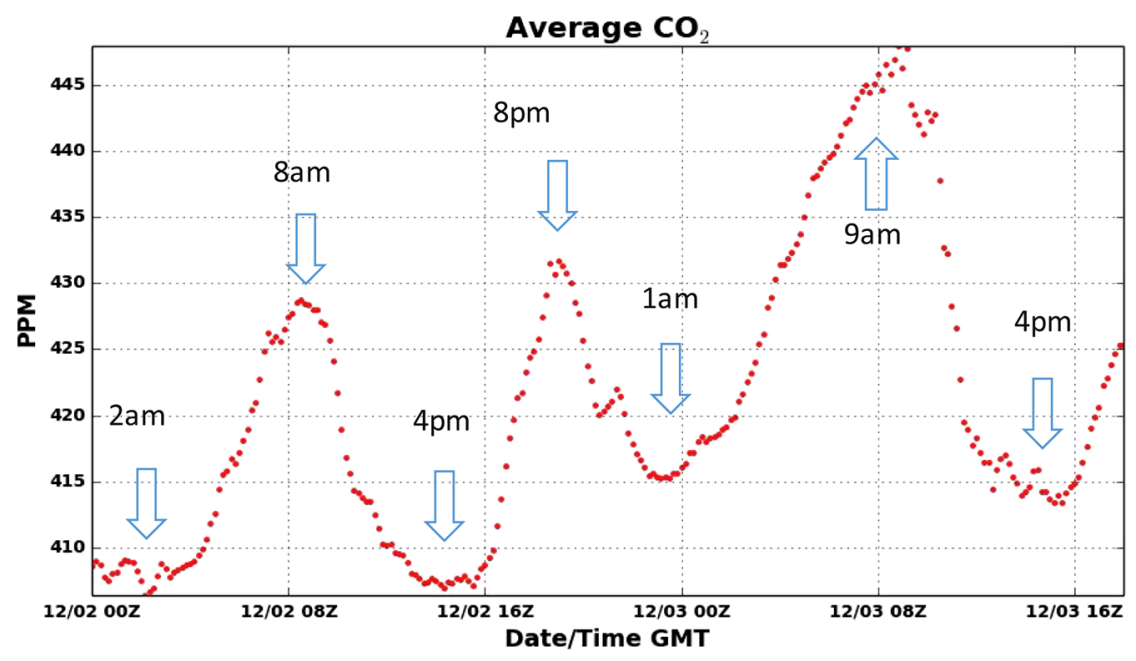

Fig. 19 Typical diurnal $\mathrm{CO}_{2}$ concentration pattern measured with the GreenLITE system in Paris.

The results shown above provide confidence in the GreenLITE system's abilities to estimate realistic $\mathrm{CO}_{2}$ concentrations that are representative of the actual present $\mathrm{CO}_{2}$ levels and to track local changes in $\mathrm{CO}_{2}$ concentrations over both short- (minutes) and long- (months) time scales.

\subsection{Reconstructing Two-Dimensional Distributions}

In addition to validating the GreenLITE dry-air mole fraction $\left(\mathrm{XCO}_{2}\right)$ column/chord retrieval mechanism, the open-air deployments described in Sec. 3 enabled the use of controlled releases of $\mathrm{CO}_{2}$ to evaluate the fidelity of the 2-D reconstruction technique and preliminary mechanisms for quantifying its performance. These efforts have included the use of both controlled aboveground and inground releases of $\mathrm{CO}_{2}$, in some cases over extended time periods. Local testing at the Harris farm test facility in New Haven, Indiana, enabled the use of aboveground releases using dual-open chambers to enhance the concentration along a pair of user-defined chords, and the ZERT facility provided a well-instrumented environment that enabled both above- and inground releases over an extended area. ${ }^{8}$

One of the challenges in modeling 2-D fields of $\mathrm{CO}_{2}$ concentrations is one's ability to crosscalibrate and/or assess the accuracy of the resulting modeled field values. While numerous methods exist for acquiring in situ point measurements of $\mathrm{CO}_{2}$ concentration as well as flux, it becomes extremely difficult to acquire enough temporally colocated data to accurately represent the distribution over an extended open path field. One can either densely sample the desired open space with numerous similar instruments or move a select set of instruments around the field of interest. Both methods are challenging at best. Having a dense network of similar instruments may be cost prohibitive and would present a calibration problem due primarily to the need to calibrate all the instruments with a common reference before deployment. Moving a single instrument or a small number of instruments around an open field assumes that the distribution is stationary over some extended period of time and that moving the instrument around does not influence the flow dynamics over the area of interest. To test the ability of the GreenLITE system to at least detect the presence of plume-like sources in a cost-effective fashion, a set of experiments was devised to enhance concentrations along some chords by placing open-ended tubes in front of a select set of reflectors (nominally two) and injecting $\mathrm{CO}_{2}$ into them to simulate intersecting chords with enhanced concentration values. Our tomographic reconstruction algorithm calculates the exact geographic location of chord intersections (to within GPS error of transceiver and retroreflector locations) and adds the retrieved concentration values of the two intersecting chords. As such, enhancing the concentration of $\mathrm{CO}_{2}$ for two known retroreflectors (chords) will simulate a source at a known intersection from the perspective of the tomographic reconstruction algorithm and serves the specific purpose of testing the reconstruction algorithm's ability to locate a source plume. The results from one such study are shown in Fig. 20. 

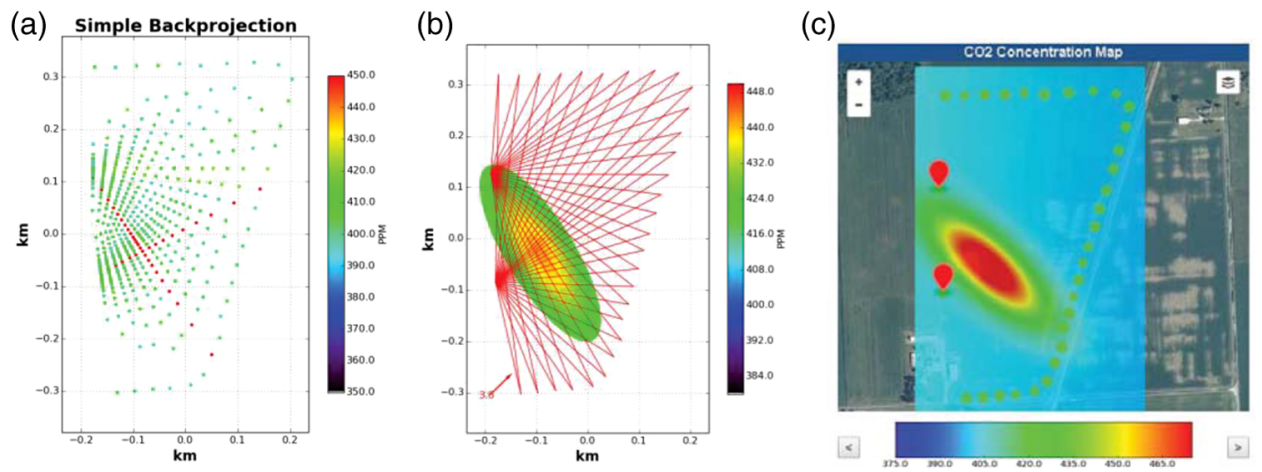

Fig. 20 Example of emulated plume data created by pumping $\mathrm{CO}_{2}$ into short tubes at the farm test site on two of the 60 possible chords. (a) Simple backprojection of all chord samples for the time period of interest and the expected chord intersections. (b) Resulting reconstruction that demonstrates system's capability to detect and locate a plume source. (c) The reconstruction as displayed on the web-based tool during the experiment, including background gradient and map.

Two chords were artificially enhanced by the placement of the open-ended tubes along T1R24 with a flow rate of $\sim 1.78 \mathrm{~m}^{3} / \mathrm{h}$ and along T2R15 with a flow rate of $\sim 1.27 \mathrm{~m}^{3} / \mathrm{h}$, where the $T$ denotes the transceiver number top to bottom and the $R$ describes the retroreflector number starting at the top left and incrementing clockwise. The retrieved values for chords T1R24 and T2R15 were $\sim 1000$ and 750 ppmv, respectively. The other retrieved values are associated with the ambient field ( 390 to 400 ppmv). Figure 20(a) shows a simple backprojection of the chord values over the sampled space. The dots represent the intersections of individual chords, and their color values represent the sum of the two retrieved concentration values at each intersection. The intersection of the two primary enhanced chords should notionally indicate the position of the source term. Figure 20(b) provides the resulting reconstructed field with a single dominant source, while Fig. 20(c) shows the reconstruction overlaid on the background concentration. The elongation is due to the greater enhancement of the chord along the NW to SE due to the higher flow rate, which elevates other intersections along that chord. While the model provides a good representation of an idealized point plume, the tests illustrate the ability of the system to detect the presence of a source in automated fashion, and in this case the model located the source to better than $11 \mathrm{~m}$ on an $\sim 0.2 \mathrm{~km}^{2}$ grid while in the presence of ambient $\mathrm{CO}_{2}$ concentrations and prevailing local wind.

Figure 21 provides a representative set of reconstructions for the ZERT installation. The green dots in each panel denote the locations of the reflectors, and the red dots indicate the geographic locations of the transceivers. The predominant feature in the upper right corner of each reconstruction correlates well with the presence of a manure pile just off to the right of the upper corner of the reconstructed field. The secondary, more diffuse field corresponds reasonably well to an extended underground controlled release in the lower left quadrant of the field inscribed by the reflectors. Additionally, Fig. 21 shows the 2-D reconstruction's ability to adapt to changes in background behavior. The ZERT reconstructions shown in this set of panels follow a portion of the time sequence shown in Fig. 12. The reconstructed images shown in the figure are centered in time near the observed spike at dusk (2:15 to 2:30 GMT) in Fig. 12 and show how the reconstruction model tracks changes in background level while still preserving persistent $\mathrm{CO}_{2}$ source features.

Finally, 2-D reconstructions of $\mathrm{CO}_{2}$ concentration fields have been continuously estimated in an urban environment since the deployment of GreenLITE in Paris in November 2015, which is of significance in validating the reconstruction algorithm due to the expanded measurement field $\left(>25 \mathrm{~km}^{2}\right.$ ), which includes path lengths of up to $5 \mathrm{~km}$. Figure 22 shows a representative 2-D reconstruction over Paris on the afternoon of 4 December 2015. Red dots indicate the location of two transceiver instruments, and purple dots indicate the retroreflector locations. The transceiver-to-reflector lines of sight during data collection are as shown in Fig. 11. The reconstructed $\mathrm{CO}_{2}$ field shows a relatively mundane distribution of $\mathrm{CO}_{2}$ with a slightly elevated concentration near the lower right quadrant of the measurement grid. 

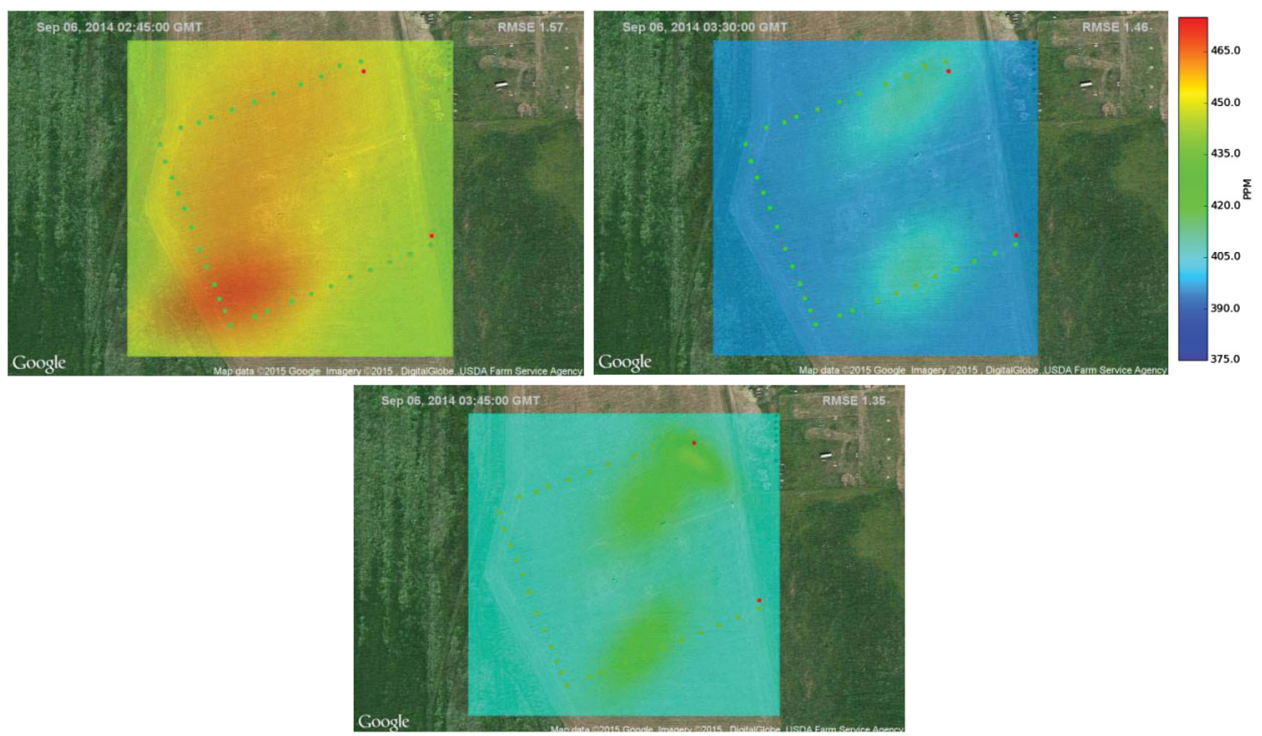

Fig. 21 Sample nighttime reconstructions based on ZERT facility test data collected over same period in time as chord values shown in Fig. 12. The retroreflector positions are denoted by the green dots in each image, and the red dots indicate the transceiver locations. The images span the time interval between 1:45 and 3:45 GMT on September 6, 2014.

(a)

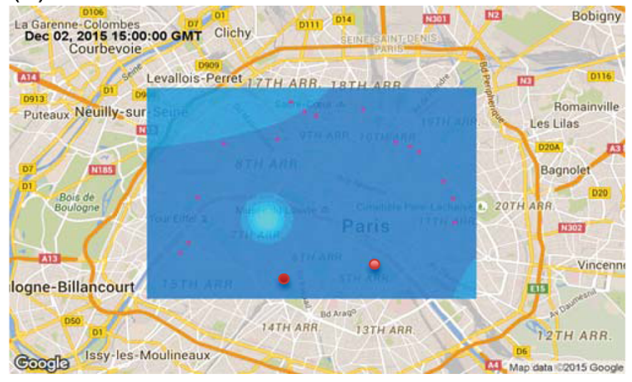

(c) (b)

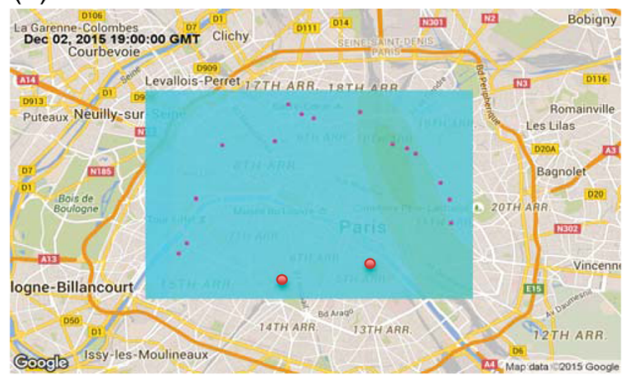

Sooglo Bobigny

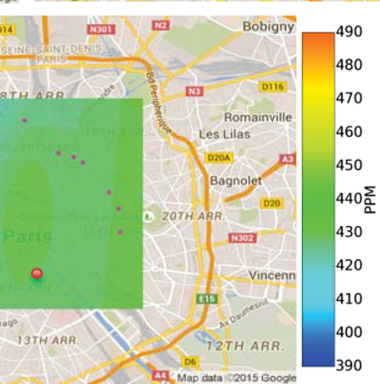

Fig. 22 2-D reconstruction fields of $\mathrm{CO}_{2}$ concentration in Paris, France. (a) December 2, 2016, 15:00 GMT. (b) December 2, 2016, 19:00 GMT. (c) December 3, 2016, 08:00 GMT.

\section{Conclusions}

A new measurement capability has been developed that can provide near-real-time information on the spatial distribution of greenhouse gases over geographical areas significant for a number of applications. Field tests have shown that the GreenLITE system measured average integrated $\mathrm{CO}_{2}$ concentrations that agreed with an independent LI-COR-based point measurement system in the field of regard of GreenLITE to within a 0.933 -correlation coefficient over several days and as high as 0.98 over one $23 \mathrm{~h}$ continuous period, despite the LI-COR instrument measuring at only a single point within the GreenLITE integrated path measurement footprint. At the ZERT 
site, signatures were clearly identified from both the buried pipeline during release rates as low as 0.2 ton/day, as well as a large natural source from decomposition of a manure pile. Measurement of a controlled release of $\mathrm{CO}_{2}$ along known chords during a field test, effectively simulating a point source at a known location, resulted in a source detection located to better than $11 \mathrm{~m}$ on a $0.2-\mathrm{km}^{2}$ grid while in the presence of ambient $\mathrm{CO}_{2}$ concentrations and prevailing local wind. The field tests and operational deployments described here demonstrate GreenLITE's ability to measure $\mathrm{CO}_{2}$ concentrations and detect/locate $\mathrm{CO}_{2}$ sources over an extended geographic area. The error in the 2-D estimate not only is dependent on the instrument measurement errors but also is a function of the site configuration. Primarily, the uncertainty is affected by the instrument accuracy, noise, the number of chords, the geometry, and the area being covered. Future efforts will be directed at determining the system's minimum detectability and attributing persistent features to man-made versus natural sources, as well as designing calibration experiments to better quantify the accuracy of measurements and the 2-D estimates.

The initial GreenLITE system was developed to be part of a monitoring and reporting system required for verification of GCS site integrity. The system was developed to measure chords over an area up to $\sim 1 \mathrm{~km}^{2}$ and generates near-real-time 2-D estimates of the spatial distribution within that area. Using the 1-km chord system as a baseline, a new 5-km chord system was built in 2015 . The $5-\mathrm{km}$ system was initially tested at the BAO tower near Boulder, Colorado, and showed a $\sim 5$ ppmv difference on average from multiple collections between the $5-\mathrm{km}$ chord data and the in situ data on the tower, with a precision of $\sim 0.5$ ppmv. The 5-km system was then deployed over the city of Paris in November 2015. Comparisons have been made with the Paris system, which show excellent agreement between the GreenLITE system and a nearby Picarro in situ instrument—again, despite GreenLITE measuring integrated path concentration versus the Picarro's point measurements-as well as a regional model run by LSCE using up- and down-wind Picarro instruments to constrain the model. The current plan is to keep the system operational through mid-November 2016, to have a full seasonal data cycle and assimilate this extensive dataset into the existing inverse transport modeling system for the estimate of the Paris emissions, which presently assimilates point measurements at perimeter-urban sites only. ${ }^{2,26}$ The assimilation of the GreenLITE urban data should help analyze the spatial distribution of emissions within Paris while the present inversions have to focus on citywide budgets due to the difficulties of exploiting urban point measurements. As mentioned above, the data from the GreenLITE system clearly show daily cycles related to natural diurnal processes and to human activities, such as traffic.

Initial comparisons with independent in situ measurements indicate that the GreenLITE system accurately tracks the $\mathrm{CO}_{2}$ concentrations within an urban environment. Although the in situ measurements used in comparisons were within or near the GreenLITE measurement footprint, they do not measure the same air mass per se and, therefore, provide only a rough estimate of systematic biases at best. With that in mind, a limited comparison to the Thermo Fisher Delta Ray showed a delta of $<1$ ppmv versus GreenLITE measurements over the 5 weeks that it operated, while the comparison to the CDS Picarro showed a delta of $\sim 6$ ppmv to GreenLITE over the same period. It also showed that during the overlapping period of the two in situ measurements they differed from each other by $\sim \pm 10 \mathrm{ppmv}$, demonstrating the challenge of evaluating absolute accuracy when measuring different air masses within an urban environment. We are in the process of planning further, more constrained, validation experiments to determine the absolute accuracy of the retrieved $\mathrm{CO}_{2}$ values. The GreenLITE system may enable measurements on the scales that models are currently capable of ingesting and could have significant impact for future predictive model capabilities. Other implementations are also being considered for providing vertical as well as horizontal mapping capabilities. Vertical transport is a large source of errors in current modeling systems, and a GreenLITE-like measurement of vertical distributions over time could add much needed information in this area.

The expansion to methane and potentially both $\mathrm{CO}_{2}$ and $\mathrm{CH}_{4}$ from a single system could significantly enhance our ability to understand complex dynamics in environments, such as cities and oil and gas production fields. We are continuing to work with our partners at LSCE and Laboratoire Atmosphères, Milieux, Observations Spatiales (LATMOS) to better validate the GreenLITE measurements, as well as evaluating the ingestion of GreenLITE data directly 
into the inversions. Initial results look very promising, and more details on this will be published soon.

\section{Acknowledgments}

We would like to acknowledge the Department of Energy, National Energy Technology Laboratory for cofunding the initial GreenLITE development under contract number DEFE002574 and the National Institute of Standards and Technology for partially funding the testing of the $5-\mathrm{km}$ system prior to deployment to Paris, France, under contract number 70NANB15H317. We would like to thank Laura Dobeck and Lee Spangler for supporting testing of the initial system at the ZERT site, and the ISGS team (specifically, Randall Locke, Curt Blakley, Carl Carman, and Sallie Greenberg) for supporting deployment of the system to the IBDP site, as well as Steve McDonald and ADM for allowing access to the site for testing the operational capabilities of the system. Thanks to Dan Wolfe and Arlyn Andrews of NOAA for support in installing the retroreflectors at the BAO tower, and to Michael Hannigan and Johanna Gordon for access to the University of Colorado Picarro data. Thanks to our contractors in Paris, Enviroearth, for their amazing work in preparing the locations for the transceivers and reflectors, as well as initial installation and continuing support. In addition, we would like to acknowledge our colleagues at Le Laboratoire des Sciences du Climat et de l'Environnement (LSCE), LATMOS, and Cité des Sciences et de l'Industrie (CDS) (Marc Jamous, Jean-Christophe Theisen, Didier Philippe, Michel Maintenant, Sylvain Aulombard, and Michel Pérez), and the staff from LSCE who helped to set up the analyzer (M. Ramonet, M. Delmotte, and M. de Florinier) for providing support and data from their measurements as comparisons with the GreenLITE data and for continuing with further modeling and comparison activities. Finally, thanks to LATMOS at University of Pierre Marie CURIE, Paris Habitat, Elogie and Montparnasse ICADE for providing the locations to install the transceivers and reflectors in Paris, France.

\section{References}

1. DOE/NETL, "Best practices for monitoring, verification, and accounting of $\mathrm{CO}_{2}$ stored in deep geologic formations-2012 update," 2012, http://www.netl.doe.gov/File\%20Library/ Research/Carbon-Storage/Project-Portfolio/BPM-MVA-2012.pdf (15 February 2015).

2. F. Bréon et al., "An attempt at estimating Paris area $\mathrm{CO}_{2}$ emissions from atmospheric concentration measurements," Atmos. Chem. Phys. 15, 1707-1724 (2015).

3. G. Burba and D. Anderson, "Introduction to the eddy covariance method general guidelines, and conventional workflow," 2005, http://www.instrumentalia.com.ar/pdf/Invernadero.pdf (10 November 2016).

4. Campbell Scientific, "Open-Path Eddy-Covariance System Operator's Manual IRGASON, KH20, and FW05," http://s.campbellsci.com/documents/us/manuals/opecsystem.pdf (April 2013).

5. J. L. Lewicki and G. E. Hilley, "Eddy covariance mapping and quantification of surface $\mathrm{CO}_{2}$ leakage fluxes," Geophys. Res. Lett. 36, L21802 (2009).

6. Z. H. Levine et al., "The detection of carbon dioxide leaks using quasi-tomographic laser absorption spectroscopy measurements in variable wind," Atmos. Meas. Tech. 9(4), 16271636 (2016).

7. W. Johnson, K. S. Repasky, and J. L. Carlsten, "Micropulse differential absorption LiDAR for identification of carbon sequestration site leakage," Appl. Opt. 52, 2994-3003 (2013).

8. L. H. Spangler et al., "A shallow subsurface controlled release facility in Bozeman, Montana, USA, for testing near surface $\mathrm{CO}_{2}$ detection techniques and transport models," Environ. Earth Sci. 60(2), 27-239 (2010).

9. S. D. Humphries et al., "Testing carbon sequestration site monitor instruments using a controlled carbon dioxide release facility," Appl. Opt. 47, 548-555 (2008).

10. K. J. Davis et al., "Greenhouse gas emissions derived from regional measurement networks and atmospheric inversions: results from the MCI and INFLUX experiments," in Proc. AGU Fall Meeting, San Francisco (2010). 
Dobler et al.: Demonstration of spatial greenhouse gas mapping using laser absorption...

11. J. Turnbull et al., "Towards quantification and source sector identification of fossil fuel $\mathrm{CO}_{2}$ emissions from an urban area: results from the INFLUX experiment," J. Geophys. Res. Atmos. 120, 292-312 (2014).

12. J. T. Dobler et al., "Atmospheric $\mathrm{CO}_{2}$ column measurements with an airborne intensity-modulated continuous wave 1.57 um fiber laser LiDAR," Appl. Opt. 52(12), 2874-2892 (2013).

13. M. E. Dobbs et al., "A sinusoidal modulated CW integrated path differential absorption LiDAR for mapping sources and sinks of carbon dioxide from space," in Proc. 14th Coherent Laser Radar Conf., Snowmass, Colorado (2007).

14. M. E. Dobbs et al., "A modulated CW fiber laser-LiDAR suite for the ASCENDS mission," in Proc. 24th Int. Laser Radar Conf., Boulder, Colorado (2008).

15. J. T. Dobler, "A novel approach for active measurement of atmospheric greenhouse gases from a geostationary orbit," in Proc. 93rd American Meteorological Society Annual Meeting, Austin, Texas (2013).

16. R. Measures, Laser Remote Sensing: Fundamentals and Applications, Wiley, New York, New York (1984).

17. S. A. Clough et al., "Atmospheric radiative transfer modeling: a summary of the AER codes, short communication," J. Quantum Spectrosc. Radiat. Transfer 91, 233-244 (2005).

18. L. Rothman et al., "The HITRAN 2008 molecular spectroscopic database," J. Quantum Spectrosc. Radiat. Transfer 110(9-10), 533-572 (2009).

19. V. M. Devi et al., "Line mixing and speed dependence in $\mathrm{CO}_{2}$ at $6227.9 \mathrm{~cm}-1$ : constrained multispectrum analysis of intensities and line shapes in the $30013 \leftarrow 00001$ band," J. Mol. Spectrosc. 245(1), 52-80 (2007).

20. V. M. Devi et al., "Line mixing and speed dependence in $\mathrm{CO} 2$ at $6348 \mathrm{~cm}-1$ : Positions, intensities, and air- and self-broadening derived with constrained multispectrum analysis," J. Mol. Spectrosc. 242(2), 90-117 (2007).

21. T. Zaccheo et al., "Impact of atmospheric state uncertainties on retrieved $\mathrm{XCO}_{2}$ columns from laser differential absorption spectroscopy measurements," J. Appl. Remote Sens. 8(1), 083575 (2014).

22. F. Cuccoli, L. Facheris, and S. Gori, "Radio base network and tomographic processing for real time estimation of the rainfall rate fields," in Proc. IEEE Geoscience and Remote Sensing Symp. (2009).

23. D. Giuli, L. Facheris, and S. Tanelli, "Microwave tomographic inversion technique based on a stochastic approach for rainfall fields monitoring," IEEE Trans. Geosci. Remote Sens. 37, 2536-2555 (1999).

24. D. Giuli et al., "Tomographic reconstruction of rainfall fields through microwave attenuation measurements," J. Appl. Meteorol. 30, 1323-1340 (1991).

25. L. Spangler, "Montana State University shallow release experiments," in Proc. Carbon Sequestration Leadership Forum, Carbon Dioxide Monitoring Interactive Workshop (2013).

26. J. Staufer et al., "A first year-long estimate of the Paris region fossil fuel $\mathrm{CO}_{2}$ emissions based on atmospheric inversion," Atmos. Chem. Phys. D 2016, 1-34 (2016).

Jeremy T. Dobler received his BS degree in physics from the University of Wisconsin-La Crosse in 1999 and his PhD in optical sciences from the University of Arizona in 2005. He is a chief engineer in the Environmental Solutions Group at Harris Corporation, where he has led a number of airborne remote sensing campaigns and several research and development efforts. His work focuses on atmospheric remote sensing instrument development/deployment and advancing available laser technologies.

T. Scott Zaccheo received his BSEE degree in from Lehigh University in 1984 and his MS degree and $\mathrm{PhD}$ in electrical engineering from Tufts University in 1993 and 1995, respectively. He serves as an AER senior vice president and the AER chief software engineer for the NOAA/ NASA GOES-R Ground System program. Over his 25+ year career, he has pursued the application of engineering principles to various atmospheric and remote sensing problems.

Timothy G. Pernini received his BA degree in physics from Rhode Island College in 2000. He is a staff scientist at AER. His work experience includes analysis of remote sensing data and 
radiometric calibration of spaced-based electro-optical sensors. He recently served as AER's lead systems engineer for implementation of the solar ultraviolet imager and magnetometer ground processing algorithms for the GOES-R weather satellite program.

Nathan Blume is a lead systems engineer in the Environmental Solutions Group at Harris Corporation, where he has been involved in the development of several active remote sensing instruments and systems over the past 13 years. He enjoys the challenges and rewards of research and development of new LIDAR technology and applications for that technology.

Gregoire Broquet has obtained his $\mathrm{PhD}$ in ocean data assimilation in 2003. Since 2009, he has supervised LSCE activities for the atmospheric inverse modeling of global greenhouse gas (GHG) fluxes from the continental to the city/industrial site scales, based on the assimilation of concentration measurements. He has coordinated the scientific activities of the industrial chair BridGES (UVSQ, CEA, CNRS, Thales Alenia Space, Veolia) dedicated to this research area.

Felix Vogel has been conducting atmospheric GHG measurements and data analysis (cosigned 22 rank A publications) since 2010. He leads efforts on city-wide GHG monitoring in Recife, Brazil, and Toronto, Canada, as well as the development and testing of lower-cost GHG sensors. From 2013 to 2016, he also coordinated the LSCE efforts on GHG monitoring for industrial sites. He is a core-team member of the WMO integrated GHG information system.

Michel Ramonet is the scientific coordinator of the French monitoring network for GHGs, which includes 16 high surface stations in France, Europe, the Indian Ocean, and South America. He was the PI of work packages from several European projects (Carboeurope, IMECC, ICOS$\mathrm{PP}$ ) and is currently the editor of the GHGs validation reports of the CAMS near-real-time global atmospheric composition service. He has published more than 100 peer-reviewed publications in A-ranking international journals.

Michael Braun is currently a senior engineer in the Environmental Solutions Group at Harris Corporation, where he has been involved in space mission electronics, spacecraft ground testing, and active remote sensing for over 30 years. His research interests include LIDAR, electro-optical instrumentation, new system design, and deployment.

Johannes Staufer received his MS degree in meteorology from the University of Vienna and his $\mathrm{PhD}$ in environmental system science from ETH Zurich. He is currently a postdoctoral fellow at LSCE, where he works mainly on estimating urban GHG emissions from concentration measurements.

Philippe Ciais obtained his PhD from the University of Paris in 1991 and was hired after a postdoc in the United States at LSCE in 1994. He is a carbon cycle researcher. He is the author of 498 publications in peer-reviewed journals on different aspects of the carbon cycle and acted as convening lead author of the fifth IPCC report.

Chris Botos received his $\mathrm{AB}$ in biology/physics from Indiana University, MSEE from Northwestern University, and worked for 36 years as an electrical and software engineer and project manager on various government and commercial electro-optical/mechanical, image and signal processing systems. He worked at AER for six years before retiring in 2016. 